\title{
Perturbation of Wound Healing, Cytoskeletal Organization and Cellular Protein Networks during Hazara Virus Infection
}

\author{
Andrea Molinas ${ }^{1+}$, Maria V. Turkina ${ }^{1+}$, Karl-Eric Magnusson ${ }^{1}$, Ali Mirazimi ${ }^{2}$ and \\ Elena Vikström ${ }^{1 *}$
}

1 Department of Clinical and Experimental Medicine, Faculty of Medicine and Health Sciences, Linköping University, Linköping, Sweden, ${ }^{2}$ Department of Laboratory Medicine, Karolinska Institute, Stockholm, Sweden

OPEN ACCESS

Edited by:

Vladimir Sytnyk,

University of New South Wales,

Australia

Reviewed by:

Thomas Fath

University of New South Wales,

Australia

Saurabh Chattopadhyay,

University of Toledo, United States

*Correspondence:

Elena Vikström

elena.vikstrom@liu.se

${ }^{\dagger}$ These authors have contributed equally to this work.

Specialty section

This article was submitted to Cell Adhesion and Migration,

a section of the journal

Frontiers in Cell and Developmental

Biology

Received: 04 July 2017 Accepted: 06 November 2017 Published: 21 November 2017

Citation:

Molinas A, Turkina MV, Magnusson K-E, Mirazimi A and Vikström E (2017) Perturbation of Wound Healing, Cytoskeletal Organization and Cellular Protein Networks during Hazara Virus Infection. Front. Cell Dev. Biol. 5:98 doi: 10.3389/fcell.2017.00098
Normal epithelial and endothelial renewal and healing after bacterial and viral challenges are essential for homeostasis along the intestine and the blood and lymphatic vessels. We thus investigated whether and how virus affects migration of human epithelial cells and specifically how the nucleocapsid protein $(\mathrm{N})$ modulates the cellular proteome and interactome using human Caco-2 cells in a wound-healing assay with Hazara virus as a model. Here, Hazara virus blocked cell migration in a dose- and time-dependent manner, disrupted the actin cytoskeleton and specifically reduced the expression of the IQ-motif-containing GTPase-activating protein 1 (IQGAP1) and water channel aquaporin 6 (AQP6) that regulate cytoskeletal organization, water homeostasis and vesicle communication. Moreover, in the Caco-2 cell proteome, we identified several distinct groups of molecules associating with $\mathrm{N}$ upon Hazara virus infection, being involved in the ensemble of important cellular processes, e.g., chaperone activity, metabolism, cellular defense against infections, cell morphology, and migration. These events do not only facilitate the virus life cycle, but they are also crucial for membrane and cytoskeleton dynamics, cellular self-renewal and wound healing, being so essential for body integrity and homeostasis.

Keywords: epithelial barrier, homeostasis, epithelial migration, aquaporin 6, IQGAP1, cellular proteome, virus infection

\section{INTRODUCTION}

Epithelial cells are positioned strategically to provide barriers to pathogens and other environmental agents. They are located both on the outside, e.g., in the skin surface, and on the inside, e.g., along the gastrointestinal tract (Ivanov et al., 2010). The linings of blood and lymphatic vessels of circulatory system are accordingly covered by a specialized form of epithelium, the endothelium (Rodrigues and Granger, 2015). These barriers display both physical and immune characteristics, where the former are potentiated by epithelial cell-to-cell junctions that prevent passage of pathogens and large molecules, and the latter are maintained via detection of antigens and recruitment for instance of phagocytes to the site of infection, potentially resulting in inflammation and tissue damage (Figure 1; Condeelis and Pollard, 2006; Ivanov et al., 2010; Rodrigues and Granger, 2015). The epithelial cell barrier consists of a monolayer of cells that are constantly moving and renewed normally every $72 \mathrm{~h}$ in the gut. This is controlled by a highly sophisticated interplay between the cytoskeleton, intercellular junctions, extracellular matrix, 

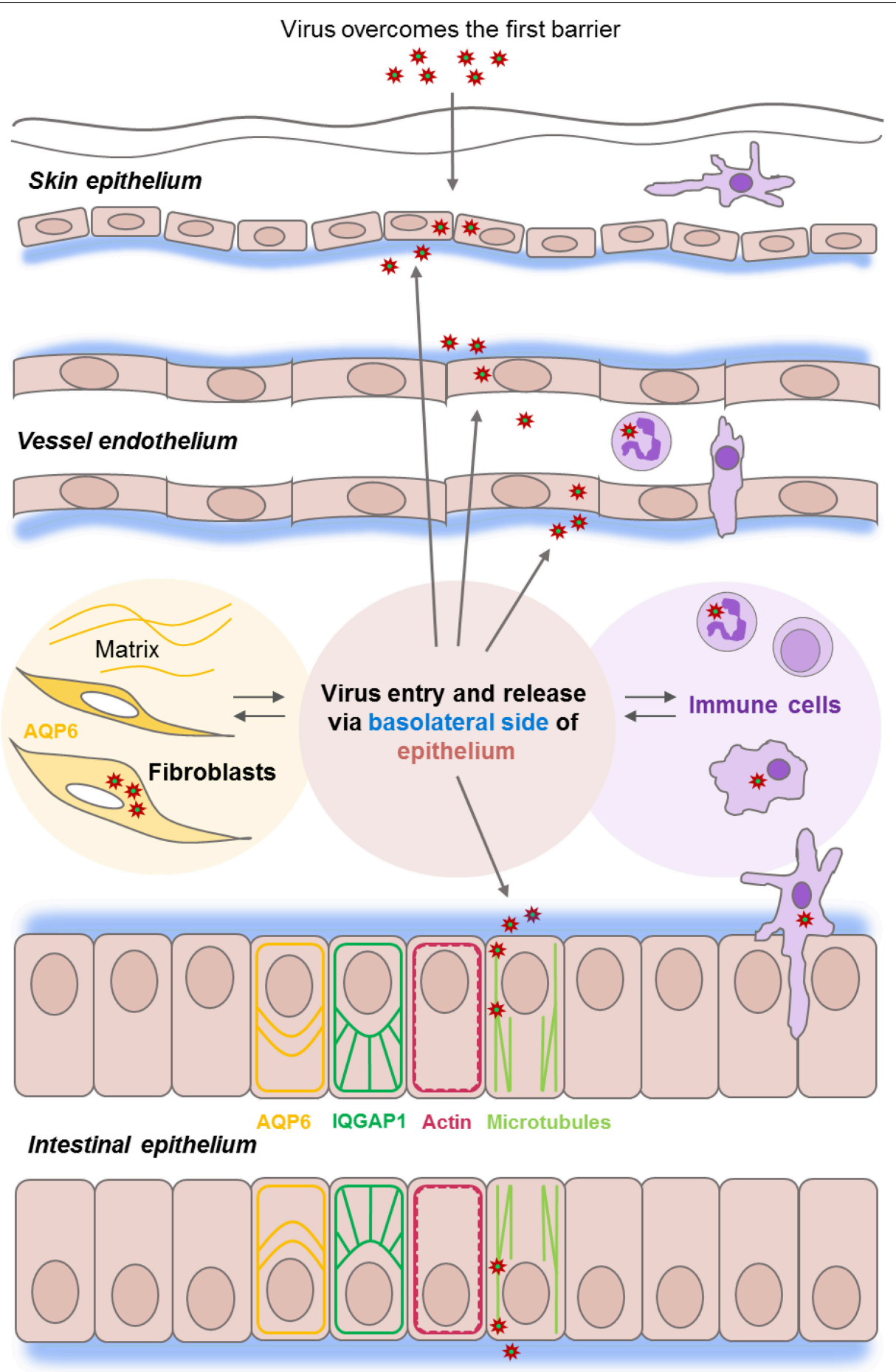

FIGURE 1 | Epithelial barrier functions at the front line of viral infections. Epithelial and endothelial cell monolayers (in pink) provide a series of host barriers to pathogens and other environmental agents. These possess both physical and immune (in lilac) properties. Epithelium constantly moving, renewing, and normally undergoes a wound-healing process which is controlled by an interplay between matrix, water homeostasis (in yellow), and cytoskeleton and junctions-associated proteins (ex. IQGAP1 in green). Viruses of the Nairovirus genus (red asterisk) can overcome the first host barrier to enter the body and access underlying cells. Viruses enter host cells by endocytosis, replicates, and assembles in the perinuclear regions of the cytoplasm and leaves the epithelial cell monolayers from the basolateral side (in blue), and further utilize microtubule (light green) and actin (red), the components of host cytoskeleton during the whole life cycle. 
surface receptors, signal mediators and fluxes of solutes and water (Ivanov et al., 2010; Rodrigues and Granger, 2015; Friedl and Mayor, 2017). Extensive remodeling of the cytoskeleton is regulated by the Rho family of small GTPases (Zegers and Friedl, 2014), where the IQ-motif-containing GTPase-activating proteins IQGAP scaffolds a plethora molecules to control diverse cellular processes, including cytoskeletal dynamics, cell migration, cell proliferation and vesicle trafficking (Karlsson et al., 2012; Hedman et al., 2015). We have thus provided an evidence that water fluxes through aquaporins (AQP) play a pivotal role in cell migration (Loitto et al., 2009; Karlsson et al., 2013), besides selectively facilitating the transport of water and small uncharged solutes like glycerol both over the cell membrane (Verkman, 2005; Benga, 2012) and intracellular membranes (Molinas et al., 2016). By such interplay with the cytoskeleton and signaling cascades, the AQP do assist directly and indirectly distinct processes, such as cell volume, signal transduction, metabolism, cell migration, and organelle physiology (Saadoun et al., 2005; Verkman, 2005; Loitto et al., 2009; Karlsson et al., 2013; Holm et al., 2016; Molinas et al., 2016).

Viruses can be inhaled or ingested as free viruses, be contained in droplets shed from an infected host, or be injected via arthropods. When they have overcome the first host barrier and enter the body, they get access to underlying permissive cells and can establish an infection (Figure 1).

Some of these arthropod-borne viruses belong to the Nairovirus genus of the Bunyaviridae family and include pathogens that cause infectious diseases in both animals and humans. The most well-known among them, Crimean-Congo hemorrhagic fever virus (CCHFV) is a human high-fatality rate pathogen causing fever, hemorrhagic symptoms and gastrointestinal disorders, such as nausea, vomiting, and diarrhea (Ergonul, 2012; Bente et al., 2013). It is an enveloped virus, with three negative-stranded RNA, designated as small, medium and large, which encode the nucleocapsid protein $(\mathrm{N})$, surface glycoproteins, and a RNA-dependent polymerase, respectively (Morikawa et al., 2007). The $\mathrm{N}$ is a multifunctional structural protein that plays a key role in the biology of RNA viruses, as it binds to the viral RNA genome and forms a ribonucleoprotein in mature virions. It also interacts with host cell proteins to facilitate the virus life cycle (Wulan et al., 2015), and localizes in the perinuclear region (Andersson et al., 2004) and in the nuclei of infected cells (Cohen et al., 2011). Since $\mathrm{N}$ is present in high amounts early during infection, it is useful for clinical diagnosis (Dowall et al., 2012b).

Working with the extremely pathogenic CCHFV requires a high biosafety level 4 laboratory, which has limited the research on specific virus-host interactions and development of antiviral therapies (Whitehouse, 2004; Dowall et al., 2015). The related Hazara virus is classified as a hazard group 2 pathogen and it is not associated with serious diseases in humans, although infections of interferon-knockout mice with either Hazara virus or CCHFV result in similar disease progression (Dowall et al., 2012a). Hazara virus and CCHFV exhibit about 70 and $86 \%$ nucleotide and amino acid sequence similarity, respectively (Honig et al., 2004), correlating well with virus serology and pathogenicity. Hazara virus has thus been used as a valid alternative experimental model for a CCHFV infection (Ergonul, 2006; Dowall et al., 2012a), enabling the investigation of Nairovirus and the development of antivirals without having access to a biosafety level 4 laboratory.

Our previous investigations have shown that Hazara virus and CCHFV enter host cells by endocytosis, replicate and assemble in the perinuclear regions of the cytoplasm and leave the epithelial cell from the basolateral side (Figure 1; ConnollyAndersen et al., 2007), and further utilize microtubule and actin, the key components of host cytoskeleton during the whole life cycle (Simon et al., 2009). Here, the cytoskeleton regulator and scaffold protein IQ-motif-containing GTPaseactivating protein 1 (IQGAP1) seems essential for virus virulence and completion of invasion, replication and egress in some viruses including Ebola, Moloney murine leukemia and swine fever (Hedman et al., 2015). We also showed recently that aquaporin 6 (AQP6), a water transporter and cytoskeleton interactor linked to an intracellular anion channel and involved in vesicle trafficking and sorting (Beitz et al., 2006; Nozaki et al., 2008), seems to have a protective role against Hazara virus infections (Molinas et al., 2016) (Figure 1, shown in yellow).

The aim of this study was to assess in greater detail consequences of virus-host cell interactions, focusing on whether and how the virus infection impacts wound-healing, cytoskeleton organization, IQGAP1 and AQP6 characteristics, and the cellular protein interactome associated specifically with the viral N. To achieve this, we used a Caco-2 epithelial cell migration assay, immunofluorescence imaging, immunoprecipitation, proteomics and bioinformatics. We found here that the virus load and infection duration strongly impacted on epithelial cell structure, signaling and the repair potential.

\section{MATERIALS AND METHODS}

\section{Epithelial Cell Culture}

Human epithelial colorectal adenocarcinoma Caco-2 cells (86010202 obtained directly from Sigma Aldrich, St. Louis, MO) were grown in Dulbecco's modified Eagle's medium (DMEM) supplemented with $10 \%$ heat-inactivated fetal calf serum, $100 \mathrm{U} / \mathrm{ml}$ penicillin, $100 \mu \mathrm{g} / \mathrm{ml}$ streptomycin, $1 \%$ non-essential amino acids and $2 \mathrm{mM}$ L-glutamine (Life Technologies, Grand Island, NY) at $37^{\circ} \mathrm{C}$ in $5 \% \quad \mathrm{CO}_{2}$. This was done for 7-10 days to allow the cells to become mature, differentiated and establish polarized epithelial monolayers.

\section{Virus Stock}

The stock for Hazara virus strain JC280 (GenBank accession number M86624.1) was produced in human adrenal cortex adeno carcinoma SW-13 cells (CCL-105 obtained directly from American Type Culture Collection, Manassas, VA) maintained in Leibovitz's L15 medium (L15) supplemented with 10\% heat-inactivated fetal bovine serum, $25 \mathrm{mM}$ HEPES, $100 \mathrm{U} / \mathrm{ml}$ penicillin, $100 \mu \mathrm{g} / \mathrm{ml}$ streptomycin (Life Technologies) at $37^{\circ} \mathrm{C}$ in $5 \% \mathrm{CO}_{2}$. 


\section{Virus Infection}

Caco-2 cell monolayers were starved in serum-free DMEM overnight and then infected with Hazara virus at three multiplicities of infection (MOI) $0.02,1$ and 2 for $1 \mathrm{~h}$ at $37^{\circ} \mathrm{C}$ in $5 \% \mathrm{CO}_{2}$ in DMEM supplemented with $2 \%$ heat-inactivated fetal bovine serum, $100 \mathrm{U} / \mathrm{ml}$ penicillin, $100 \mu \mathrm{g} / \mathrm{ml}$ streptomycin. After 1-h infection, cells were rinsed and maintained for 24 or $48 \mathrm{~h}$ post infection (hpi) at $37^{\circ} \mathrm{C}$ in $5 \% \mathrm{CO}_{2}$ in serum free DMEM supplemented with $100 \mathrm{U} / \mathrm{ml}$ penicillin, $100 \mu \mathrm{g} / \mathrm{ml}$ streptomycin. Then, the cells were processed for migration assay, imaging, and proteomics.

\section{Migration Assay}

Caco-2 cells were seeded in $\mu$-dishes with inserts (Ibidi GmbM, Martinsried, Germany) and cultured for 10-12 days to allow the cells to become $100 \%$ confluent, mature, differentiated and polarized epithelial monolayers. Then, cells were serum-starved overnight and either non-infected or infected with Hazara virus at MOI 0.02 and 2.0 for $1 \mathrm{~h}$ at $37^{\circ} \mathrm{C}$ in $5 \% \mathrm{CO}_{2}$ in serum free DMEM supplemented with $100 \mathrm{U} / \mathrm{ml}$ penicillin, $100 \mu \mathrm{g} / \mathrm{ml}$ streptomycin. After 1-h infection, the cells were rinsed and maintained for 0, 24, or 48 hpi (Figure 2). At 0, 24 and 48 hpi, the insert was removed to get two cell patches with a $500 \pm 50 \mu \mathrm{m}$ cell-free gap in between allowing the cells to migrate and heal the wound. For each dish, the images of cells migrating into the gap area were taken at indicated time points (Figure 2) using the benchtop microscope JuLI (NanoEnTek Inc., Seoul, South Korea); between imaging, cells were returned to the incubator. Migration activity was calculated by measuring the relative area of the image occupied by cells on each dish at each time point with the Image J software (NIH, Bethesda, MD https://imagej. nih.gov/ij/). At least 3 independent experiments were performed on separate days on different cell passages.

\section{Determination of Virus with Immunofluorescence Microscopy}

Determination of the concentration of Hazara virus in the virus stock and in the monolayers after migration experiments was performed as described previously (Andersson et al., 2004). Briefly, SW-13, or Caco-2 cells in 96-well plates were infected with 10-fold serial dilutions of the virus stock for $1 \mathrm{~h}$ at $37^{\circ} \mathrm{C}$ in $5 \% \mathrm{CO}_{2}$. Alternatively, Caco-2 monolayers in $\mu$-dishes after closure of the wound were used. Cells were fixed, permeabilized, labeled with polyclonal rabbit antiCCHFV/Hazara N antibodies (Andersson et al., 2004) and Alexa Fluor 488-conjugated polyclonal goat anti-rabbit antibodies (A11008, Life Technologies). Fluorescent foci were counted in an Axiovert 35 fluorescence microscope (Carl Zeiss, Jena, Germany) equipped with a ProgResC camera (Jenoptik, Jena, Germany). This allowed the determination of the infectious virus titer in focus forming units per $\mathrm{ml}(\mathrm{FFU} / \mathrm{ml})$.

\section{Laser Scanning Confocal Imaging}

Caco-2 monolayers grown on glass coverslips (thickness 0.17 \pm 0.01, 13 mm-diameter; Karl Hecht Assistent, Sondheim, Germany) were washed with PBS pH 7.3 and pretreated with $0.2 \%$ Triton X-100 (Sigma Aldrich) in PBS for $2 \mathrm{~min}$ on ice.
The samples were then fixed in $2.5 \%$ paraformaldehyde (Sigma Aldrich) in PBS pH 7.3 for $20 \mathrm{~min}$ at room temperature. The pre-treatment with $0.2 \%$ Triton X-100 was important for a clear labeling of proteins. After washing with PBS cells were further permeabilized in $0.05 \%$ Triton X-100 in PBS for $5 \mathrm{~min}$ and washed again. Non-specific background staining was blocked for $10 \mathrm{~min}$ in PBS containing $1 \%$ BSA and $10 \mathrm{mM}$ glycine. The washing was repeated, and polyclonal rabbit or monoclonal mouse antibodies against CCHFV/Hazara N (Andersson et al., 2004), monoclonal mouse antibodies against IQGAP1 or polyclonal rabbit antibodies against AQP6 (05-504, AB3073, Millipore, Temecula, CA), diluted 1:200 in blocking buffer were then applied for $1 \mathrm{~h}$ at $37^{\circ} \mathrm{C}$ in a moist chamber. After washing, Alexa 568-conjugated goat anti-rabbit and Alexa 488-conjugated goat anti-mouse antibodies (A11036, A11029, Life Technologies) diluted 1:400 were added and incubated for $1 \mathrm{~h}$ at $37^{\circ} \mathrm{C}$ in a moist dark chamber. To detect F-actin, cells were stained with Alexa 488-conjugated phalloidin (A12379, Life Technologies). Nuclei were labeled with DAPI (Life Technologies), according to the manufacturer's instructions. Finally, coverslips were mounted in ProLong Gold (Life Technologies). The specimens were examined through $63 x$ oil immersion objectives with NA 1.40 in a fluorescence microscope Zeiss Axio Observer Z1 with the confocal system Zeiss LSM700 and Zeiss ZEN software (Carl Zeiss, Jena, Germany). Fluorescence intensity in juxta-membrane regions in the cell monolayers and intensity profile plots across the cell monolayers were measures and quantified using the Image J software (NIH). At least 3 independent experiments were done on separate days on different cell passages.

\section{Total Cell Lysates, SDS-PAGE and Immunoblotting}

Caco-2 monolayers grown on 6-well plates or flasks were washed with PBS, pH 7.6 and lysed with ice-cold RIPA buffer $(150 \mathrm{mM}$ $\mathrm{NaCl}, 1 \%$ deoxycholic acid sodium salt, $1 \% \mathrm{~N}-40,0.1 \%$ SDS, $10 \mathrm{mM}$ EDTA $\mathrm{pH} 8.0,10 \mathrm{mM}$ Tris $\mathrm{pH} 7.4$ dissolved in PBS) supplemented with $25 \mathrm{U}$ nuclease (Thermo Scientific, Rockford, IL), $1 \mathrm{mM}$ phenyl-methyl-sulfonyl-fluoride, $1 \mathrm{mM} \mathrm{Na} \mathrm{VaO}_{4}$, $25 \mathrm{mM} \mathrm{NaF}$ (Sigma Aldrich), protein inhibitors Complete (Roche Diagnostics, Mannheim, Germany). Cell suspensions were homogenized through a 21-gauge needle and centrifuged at $18,000 \mathrm{~g}$ for $30 \mathrm{~min}$ at $4^{\circ} \mathrm{C}$, and the supernatants were collected. The protein concentration in cell lysates was measured with the Bio-Rad $\mathrm{D}_{\mathrm{C}}$ protein assay (Bio-Rad Laboratories, Hercules, CA). The samples were further diluted in Laemmli sample buffer at equal protein concentrations, heated for $5 \mathrm{~min}$ at $95^{\circ} \mathrm{C}$ and then subjected to electrophoresis. They were loaded on 8-16\% SDS-polyacrylamide gels (Lonza, Rockland, ME), and after separation, proteins were electrophoretically transferred to a PVDF Immobilon-FL membrane (Millipore); the quality of the transfer was controlled by Ponceau S staining (Sigma Aldrich). Non-specific binding was blocked by 1 -h incubation in 5\% non-fat milk in PBS, pH 7.6 containing $0.18 \%$ Tween 20 at room temperature. The membranes were then incubated with antibodies against CCHFV/Hazara N (Andersson et al., 2004), IQGAP1, AQP6, and GAPDH (05-504, AB3073, MAB374 


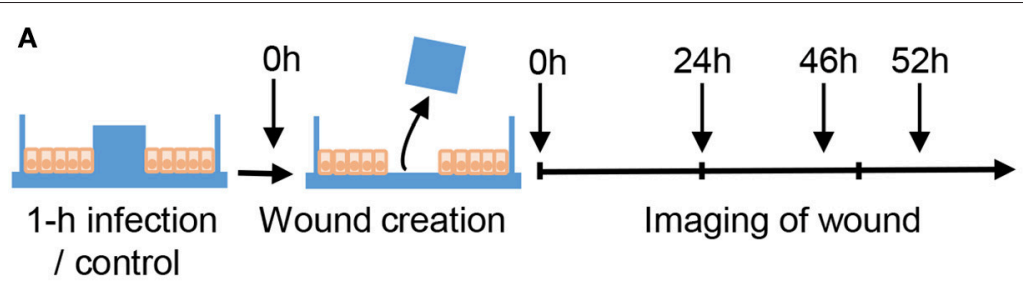

B

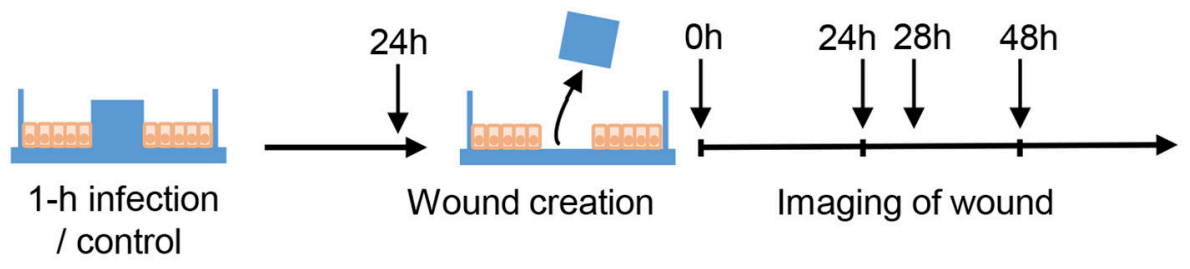

C

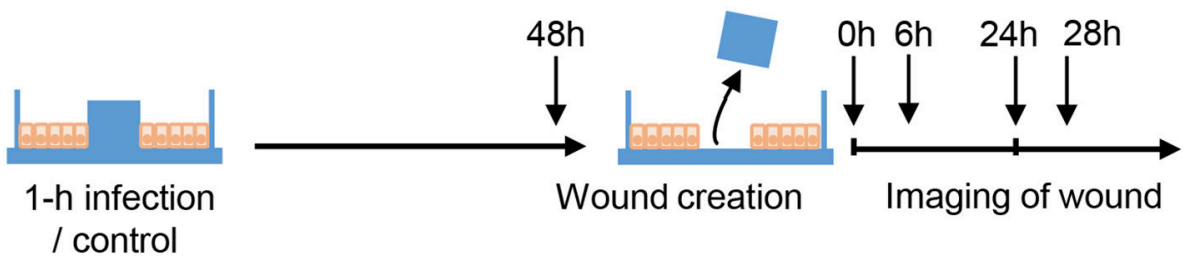

FIGURE 2 | Schematic layout of wound healing assays. Caco-2 cells were cultured in $\mu$-dishes with lbidi inserts until monolayers were confluent. Cells were then either untreated (Control) or infected with Hazara virus at different MOI for $1 \mathrm{~h}$. After 1-h infection, the cells were rinsed and maintained for 0 , 24 , or 48 hpi as shown in (A-C) respectively. To create a wound, the inserts were removed at 0, 24, or $48 \mathrm{hpi}$, and the cells were thereafter allowed to migrate. To monitor the wound healing, the images of cells migrating into the gap area of the wound were taken at indicated time using the benchtop microscope.

Millipore) diluted 1:1000 in blocking buffer overnight at $4^{\circ} \mathrm{C}$. After washing, they were treated for $1 \mathrm{~h}$ at room temperature with IRDye $800 \mathrm{CW}$ goat anti-rabbit or IRDye $680 \mathrm{CW}$ goat antimouse antibodies (926-32,211, 926-68,070, LI-COR Biosciences, Cambridge, UK), diluted 1:10,000 and washed extensively. The signals were detected and the density ratio was quantified using the Odyssey CLx and the Image Studio software (LI-COR). At least 4 independent experiments were performed on separate days on different cell passages.

\section{Immunoprecipitation}

Total-cell lysates were pre-cleared for $30 \mathrm{~min}$ at $4^{\circ} \mathrm{C}$ with of protein G-Sepharose "4 fast flow" (GE Healthcare, Uppsala, Sweden) and centrifuged before the protein concentration in the supernatants was determined with the Bio-Rad $\mathrm{D}_{\mathrm{C}}$ protein assay. Samples with equal protein concentrations were then precipitated overnight at $4^{\circ} \mathrm{C}$ with $1 \mu \mathrm{g}$ antibodies against CCHFV/Hazara N (Andersson et al., 2004). Immune complexes were captured at $4^{\circ} \mathrm{C}$ overnight using protein G-Sepharose "4 fast flow." The beads were collected by pulse centrifugation and washed three times with cold PBS, pH 7.6. Sepharose beads were re-suspended in Laemmli sample buffer, boiled for $5 \mathrm{~min}$ at $95^{\circ} \mathrm{C}$, collected by centrifugation, and the supernatant was subjected to 8-16\% SDS-PAGE (Lonza). The gels were stained with Coomassie Blue (Thermo Scientific). At least 4 independent experiments were done on separate days on different cell passages.

\section{Protein Identification by In-Gel Digestion and LC-MS/MS}

Coomassie Blue-stained protein bands were excised, reduced, alkylated and digested as described previously (Shevchenko et al., 2006); generated peptides were dried, dissolved in $0.1 \%(\mathrm{v} / \mathrm{v})$ formic acid in water and analyzed by LC-MS/MS. Peptides were separated by reverse phase chromatography on a $20 \mathrm{~mm} \times 100 \mu \mathrm{m} \mathrm{C18}$ pre column followed by a $100 \mathrm{~mm} \times$ $75 \mu \mathrm{m}$ C18 column with particle size $5 \mu \mathrm{m}$ (NanoSeparatoons, Nieuwkoop, Netherlands) at a flow rate $300 \mathrm{~nL} / \mathrm{min}$. EASYnLC II (Thermo Scientific) by gradient of $0.1 \%$ formic acid in water (A) and $0.1 \%$ formic acid in acetonitrile (B) as follows: $0-30 \% \mathrm{~B}$ in $50 \mathrm{~min}, 30-100 \% \mathrm{~B}$ in $40 \mathrm{~min}$. Automated online analyses were performed with a LTQ Orbitrap Velos Pro hybrid mass spectrometer (Thermo Scientific) with a nano-electrospray source. 


\section{Database Searching}

Raw files were analyzed using Sequest $\mathrm{HT}$ in Proteome Discoverer (Thermo Fisher Scientific, San Jose, CS version 1.4.0.288) against a Uniprot Human database available at UniProtKB website (http://www.uniprot. org/taxonomy/9606) with the following parameters: trypsin as a digestion enzyme; maximum number of missed cleavages 2; fragment ion mass tolerance $0.60 \mathrm{Da}$; parent ion mass tolerance $10.0 \mathrm{ppm}$; fixed modification, carbamidomethylation of cysteine; variable modifications, methionine oxidation.

\section{Data Evaluation and Label-Free Quantification}

Identified proteins were validated using SCAFFOLD (Version 4.4.8; Proteome Software Inc., Portland, OR). Identifications were based on a minimum of 2 unique peptides, minimum $80 \%$ peptide identification probability (using the Scaffold Local FDR algorithm), and minimum 95\% protein identification probability (using the Protein Prophet algorithm (Nesvizhskii et al., 2003), resulting in a $0.0 \%$ decoy FDR). Proteins that contained similar peptides and which could not be differentiated based on MS/MS analysis alone were grouped to satisfy the principles of parsimony. The label-free quantitative analysis of peptides was performed by spectral counting analysis using normalized spectral abundance factor (NSAF) calculated for each protein to normalize run-to-run variations (Zybailov et al., 2006), and quantitative differences were statistically analyzed by two-tailed Student's $t$-test. Differences with $P$ $<0.1$ were considered statistically significant. Identified proteins were categorized according to gene ontology terms.

\section{Bioinformatics Analysis}

The interactions between the identified cellular proteins were analyzed by the Search Tool for the Retrieval of Interacting Genes and Proteins, STRING 10.0 (http://www.ncbi.nlm. nih.gov/pubmed/18940858) using medium confidence score 0.4 and all active interaction sources (Franceschini et al., 2013). STRING and NCBI GO annotations by SCAFFOLD analyses were used to group proteins into functional classes.

\section{Statistical Analysis}

Data in the graphs are presented as mean \pm SE. Statistical analyses are based on two-tailed Student's $t$-test. The numbers $(n)$ are specified in the figure legends. $P<0.05\left(^{*}\right),<0.01$ $\left({ }^{* *}\right)$, and $<0.001\left(^{* *}\right)$ were considered significant. The experiments on migration and imaging were done at least 3 times on separate days on different cell passages. The experiments for immunoblotting, immunoprecipitation, proteome and interactome were repeated 4 times on separate days. Data evaluation and quantification for proteome and interactome experiments are described above.

\section{RESULTS}

\section{Hazara Virus Modulates Migration and Wound-Healing Capacity of Epithelial Cell Monolayers}

When viruses target and enter epithelial cells (ConnollyAndersen et al., 2007), a further perturbation of cell properties and wound-healing process may occur. Therefore, we investigated whether an infection with Hazara virus affected epithelial cell migration, using Ibidi wound-healing chambers (Figure 2). The mature differentiated and polarized epithelial monolayers were infected with Hazara virus at two multiplicities of infection (MOI), 0.02 and 2.0 for $1 \mathrm{~h}$, and wounds were then created at 0 , i.e., immediately, or at 24 and $48 \mathrm{~h}$ post infection (hpi), allowing the cells to migrate and heal the wound for up to $52 \mathrm{~h}$ (Figures 2, 3). For the 0-hpi wound, we observed that the migration rates of virus-infected cells were similar to the control, i.e., untreated cells (Figure 3A). For the wounds created at 24 hpi, the rates of healing for cells infected with Hazara virus at MOI 2.0 were significantly suppressed at $30-52 \mathrm{~h}$. By contrast, the MOI 0.02 resulted in significantly promoted migration between 24 and $30 \mathrm{~h}$ after creation of wounds, as compared to the untreated control (Figure 3B). When the wounds were created at $48 \mathrm{hpi}$, the migration rates of cells infected with Hazara virus at both MOI 0.02 and 2.0 and 0.02 were significantly inhibited around 24-28 h (Figure 3C); the control non-infected wounds were closed at that time and for this reason the study was not continued longer. In this set of experiments, no signs of Caco-2 cell death or apoptosis were seen.

To conclude, Hazara virus can perturb the normal physiology of epithelial cell monolayers and modulate their migration and wound-healing capacity in a dose- and time-dependent manner.

\section{Hazara Virus Infection Reduces the Expression of Cytoskeleton-Associated Proteins}

Individual and collective cell migration and restitution of epithelium are driven by actin cytoskeleton reorganization, primarily by dynamic polymerization of monomeric G-actin to F-actin (Ivanov et al., 2010; Friedl and Mayor, 2017) and are regulated by an ensemble of interacting proteins, including IQGAP1 (Karlsson et al., 2012; Hedman et al., 2015) and water fluxes via AQP (Karlsson et al., 2013), which facilitate Factin formation. Using confocal imaging, we disclosed dramatic decreases in F-actin as well as redistribution of IQGAP1 and AQP6 in Caco-2 monolayers infected with Hazara virus at MOI 1 for $24 \mathrm{~h}$, in clear contrast to control cells (Figures 4A-C). The distribution of these proteins in juxta-membrane regions went from a distinct uniform to a more disorganized and diffuse pattern; AQP6 re-localized to the cytoplasm; IQGAP1 formed cytoplasmic aggregates; and this was observed in more or less all cells in different fields of views. The quantification of fluorescence intensities of F-actin, IQGAP1 and AQP6 in juxta-membrane regions revealed a pronounced and significant decrease of intensity in virus-infected cells (Figures 4D-F), suggesting that cell-to-cell contacts and junctional associations 


\section{Wound healing after 1-h infection and wound creation at:}
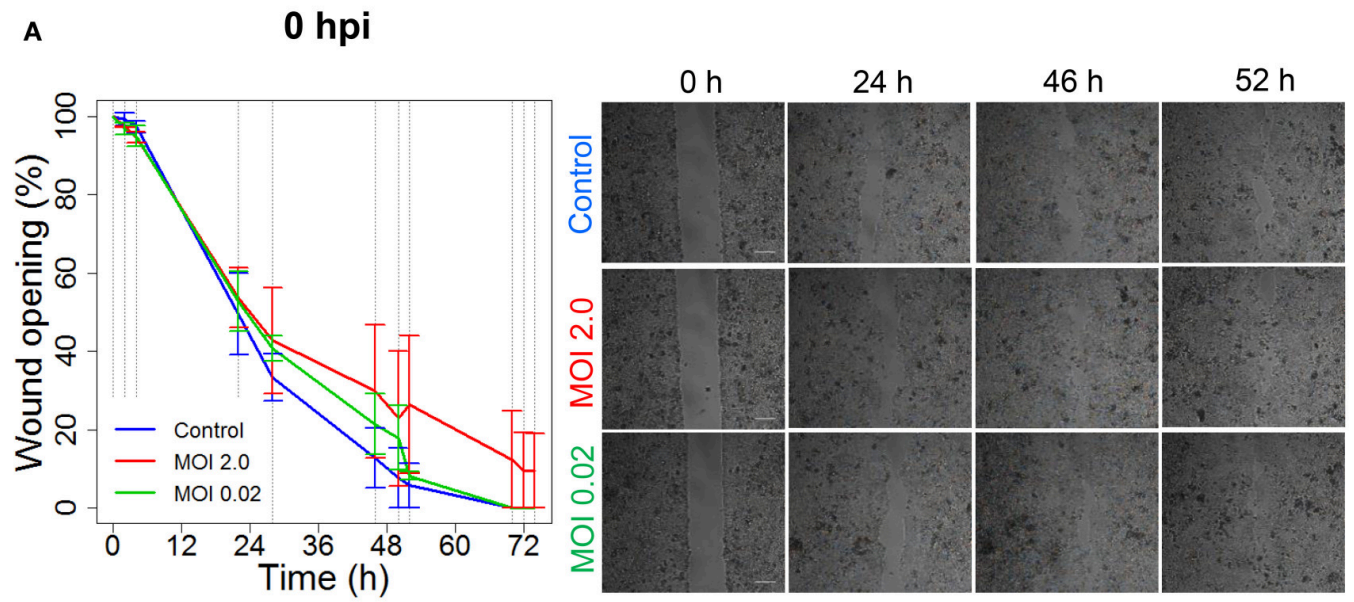

\section{B \\ 24 hpi}
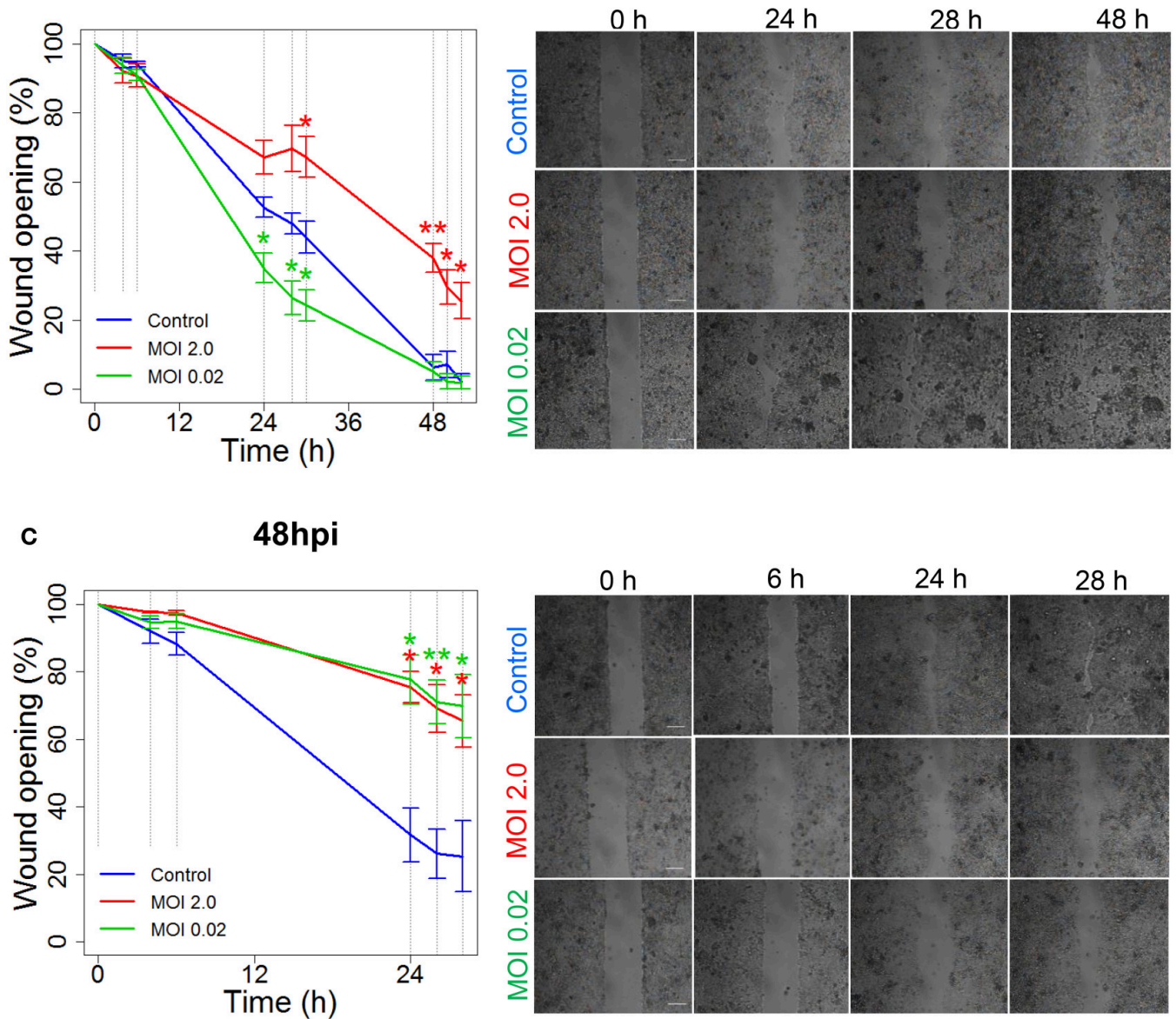

FIGURE 3 | Hazara virus modulates wound healing. Wound healing assays were performed as shown in Figure 2. Cell monolayers were either untreated (Control) or infected with Hazara virus at $\mathrm{MOI} 2.0$ and 0.02 for $1 \mathrm{~h}$. After 1-h infection, the cells were rinsed and maintained for 0, 24, or 48 hpi, as in (A-C) respectively. To create a wound, the inserts were removed at 0,24 , or 48 hpi, and the cells were thereafter allowed to migrate as shown in images in in the right panels in (A-C), respectively. The graphs in the left panels represent quantification of wound opening, shown as percent (\%) of the original gap. Values are the mean \pm SE based on three independent experiments performed on separate days from different cell passages $(n=3)$. Significant differences were analyzed by Student's two-tailed $t$-test and are indicated with * or ${ }^{\star *}$ when $P<0.05$ or $P<0.01$ compared to control. Representative images of one of three independent experiments are shown in panels on the right. Bar $200 \mu \mathrm{m}$ 


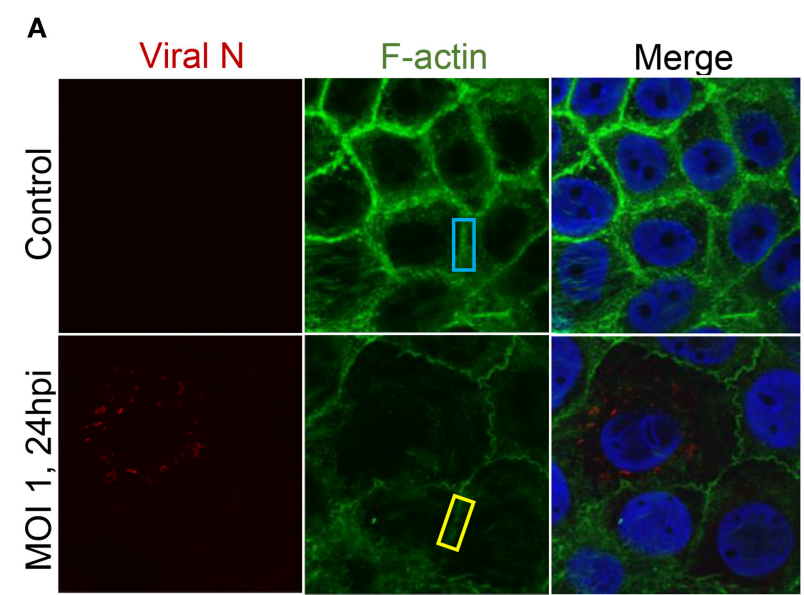

B

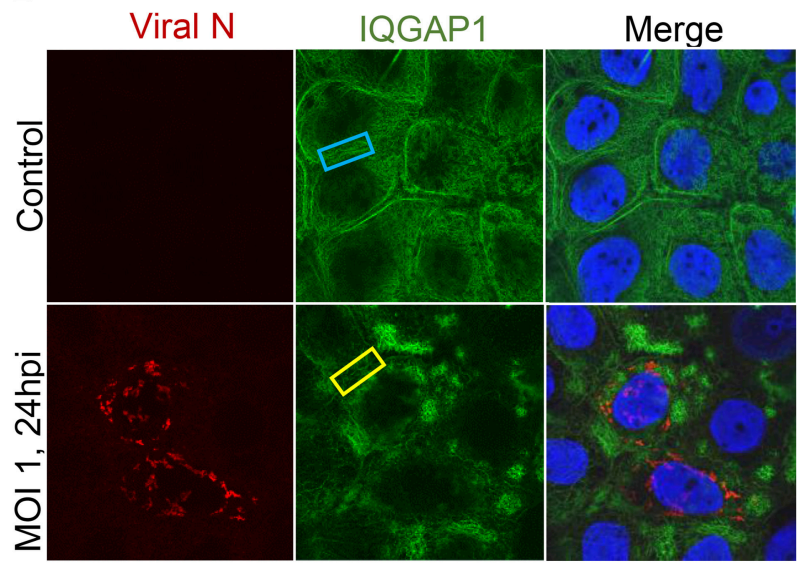

C

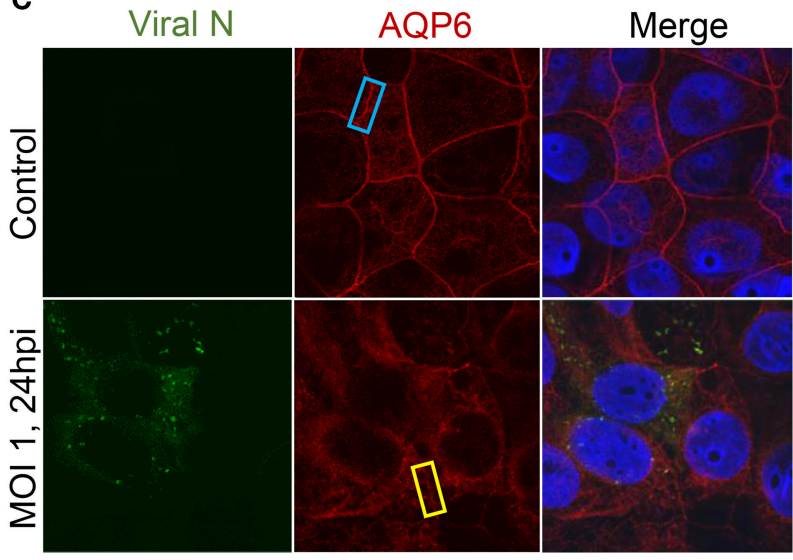

B

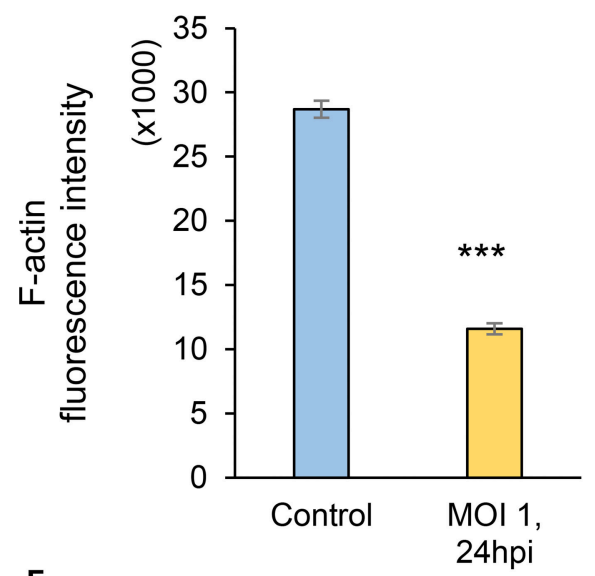

E

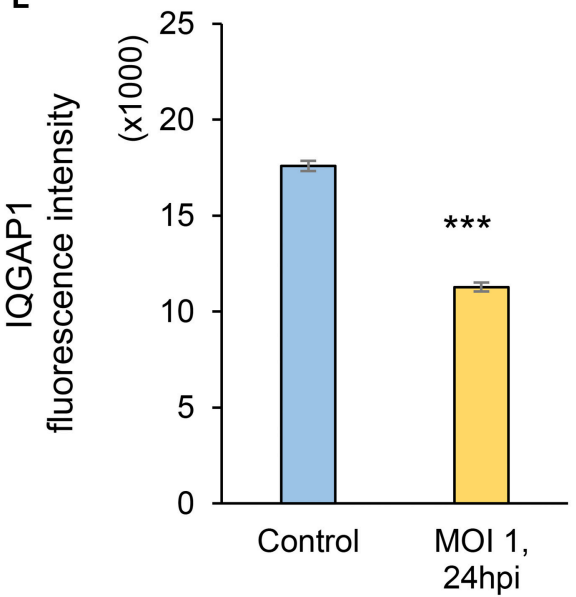

$\mathbf{F}$

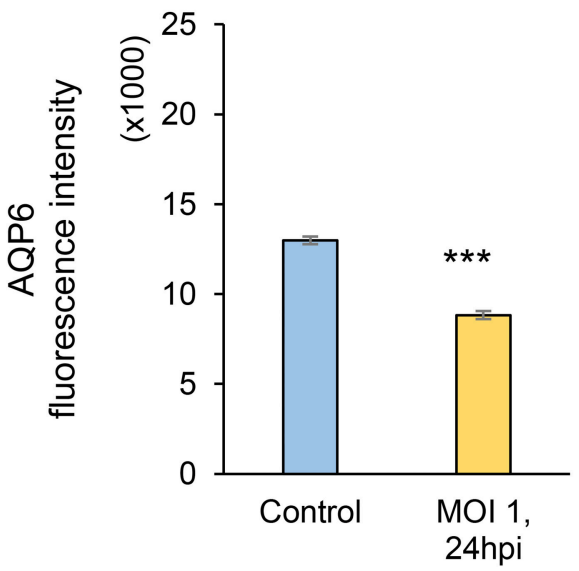

FIGURE 4 | Visualization of Hazara virus, F-actin, IQGAP1 and AQP6 in epithelial cells. Caco-2 cell monolayers were infected with Hazara virus at MOI 1 for $1 \mathrm{~h}$ and maintained for 24 hpi. Untreated non-infected Caco-2 cell monolayers were used as a control. Samples were then fixed and stained for: (A) viral N (red) and F-actin (green); (B) viral N (red) and IQGAP1 (green); (C) viral N (green) and AQP6 (red); nuclei were labeled with DAPI (blue). Samples were analyzed by confocal microscopy. The data is from one representative of three independent experiments. Image size is $67.6 \times 67.6 \mu \mathrm{m}$ and pixel size is $0.13 \mu \mathrm{m}$. Quantification of fluorescence intensities for F-actin (D), IQGAP1 (E) and AQP6 (F) measured in juxta-membrane regions in cell monolayers as indicated by blue (untreated control) and yellow rectangles (virus-infected cells, MOI 1, 24 hpi) in (A-C). Values in graphs represent the mean \pm SE based on 3 independent experiments and 60-63 cells for each condition (same color code as in images). Significant differences were analyzed by Student's $t$-test and are indicated with ${ }^{\star \star \star}$ when $P<0.001$ compared to control. 
are indeed affected. To further quantify, intensity profile plots across the cell monolayers were measured (Figures S1-S4). Here, non-infected controls displayed pronounced distinct assemblies of F-actin, IQGAP1 and AQP6 at cell-to-cell contacts, whereas the virus-infected cells had clearly more smooth profiles. Viral $\mathrm{N}$ was observed in the perinuclear region of infected cells (Figures 4A-C), which is in line with an earlier report (Andersson et al., 2004). Next, we investigated the effect of Hazara virus on the expression levels of IQGAP1 and AQP6 in epithelial cells with immunoblotting (Figure 5A) and further quantification of the density of the bands (Figures 5B,C). Here, the infection with Hazara virus at MOI 1 for $24 \mathrm{~h}$ caused a significant decrease in the expression level of either protein.

Thus, the Hazara virus infection resulted in less cytoskeletal F-actin, altered distribution and reduced expression of IQGAP1 and AQP6 appealing for perturbed organization of the cytoskeleton.

\section{Alterations in the Human Cellular Interactome of the Viral N}

Since Hazara virus challenges resulted in modified epithelial migration (Figure 3) and reduced expression of several proteins important for cytoskeletal dynamics and homeostasis (Figures 4, 5), other parallel manipulations of cellular processes and programs might be of significance. We thus further aimed to identify partners of Hazara virus nucleocapsid protein $(\mathrm{N})$ in the cellular proteome of human epithelia upon infection. To do so, epithelial monolayers were kept non-infected, or infected with Hazara virus at MOI 1 for $1 \mathrm{~h}$, and at $24 \mathrm{hpi}$, total cell lysate samples were obtained and further immunoprecipitated using the antibodies against CCHFV/Hazara N. The N-enriched immune complexes, as well as total cell lysate samples were separated on SDS-PAGE and stained with Coomassie Blue (Figure 6). For the latter, no significant difference in protein patterns of infected and non-infected cells was observed (Figure 6, right panel). For the samples with $\mathrm{N}$-enriched immune complexes, one additional protein band was reproducibly seen in the sample corresponding cells infected with Hazara virus (Figure 6, left panel).

Each gel line was cut into 10 slices, digested and analyzed by liquid chromatography and mass spectrometry (LC-MS/MS), where spectrum counts were used for quantitative comparisons of samples. To provide confidence, the experiments were repeated 4 times. The additional visible band detected in the sample corresponding cells infected with Hazara virus was identified as the viral N (Figure 6, left panel), which served as a control to confirm both infection and immunoprecipitation. Furthermore, 36 out of around 500 cellular proteins were typically identified in the $\mathrm{N}$-enriched immune complexes and exhibited significant differences in affinity (Table $\mathbf{1}$ ).

Ten $\mathrm{N}$-associated proteins were detected in virus-infected cells only, and 13 proteins displayed at least 2 -fold increased binding to $\mathrm{N}$-enriched immune complexes upon viral infection in comparison to the non-infected control (Table 1). These 23 proteins were considered as potential $\mathrm{N}$ partners in the Hazara infection. Twelve proteins displayed decreased binding to $\mathrm{N}$-enriched immune complexes upon viral infection in comparison to non-infected control (Table 1). All 36 cellular proteins, typically identified in $\mathrm{N}$-associated immune complexes and exhibited significant differences in affinity (Table 1), were included in the further bioinformatics analyses.

Bioinformatics analyses, using the search tool for the retrieval of interacting genes and proteins (STRING) and NCBI GO annotations by SCAFFOLD, were performed to investigate whether the identified $\mathrm{N}$-associated cellular proteins could be placed into distinct functional classes. This revealed that they could be put in a groups being involved in: RNA and DNA processes, formation of membrane-bound vesicles, cell morphology, migration, differentiation, proliferation and apoptosis, metabolism, cellular defense and response to stress and pathogens, virus growth and chaperone activity (Table 1).

Independent analyses of network interactions, using STRING, disclosed that out of the 36 host proteins, which were typically identified in $\mathrm{N}$-enriched immune complexes, at least 24 were connected through distinct types of actions (Figure 7), as previously being established experimentally and from curated databases and predicted by gene neighborhood and co-occurrence or by protein co-expressions and homologies (Figure S5). The distinct, large node cluster of $\mathrm{N}$-associated proteins having a rich network of protein-protein interactions (Figure 7) contained proteins with chaperone activity (Table 1, depicted in blue): calnexin, mitochondrial Hsp60 and Hsp70, stress-induced phosphoprotein 1, elongation factor $1-\delta, \alpha-$ enolase, hsc70-interacting protein and two members of the protein disulfide isomerase family. Other proteins with a rich network were ribosomal receptor of activated protein $C$ kinase 1 and cytoplasmic heterogeneous nuclear ribonucleoprotein $\mathrm{Q}$ (Figure 7). Further bioinformatics analyses revealed clusters of proteins involved in RNA and DNA processes (Table 1, noted in red, Figure S6A), in membrane-bound vesicle communication (Table 1, shown in violet, Figure S6B), and in key cellular processes, such as cell morphology, migration, differentiation, proliferation and apoptosis (Table 1, shown in green).

Thus, epithelial cellular protein networks are strongly perturbed by a Hazara virus infection, where the viral structural $\mathrm{N}$ associates and interacts with an array of cellular proteins.

\section{DISCUSSION}

A fundamental feature of epithelial linings of multicellular organisms is their ability to repair wounds via regeneration and collective cellular movement (Friedl and Mayor, 2017). Both properties are crucial for the maintenance of the structural and functional integrity and homeostasis of tissues (Figure 1). Employing the model of epithelial wound healing (Figure 2), we show that the repair was perturbed upon Hazara virus infection (Figure 3). At early stages of infection, the migration rates of virus-infected cells were either similar to the control or even promoted (Figures 3A,B). This probably reflects the very short time for establishment of infection. Indeed, in CCHFV, RNA synthesis in the infected cells likely starts at 6-16 h after infection (Simon et al., 2009). Later, we observed consistently significantly 


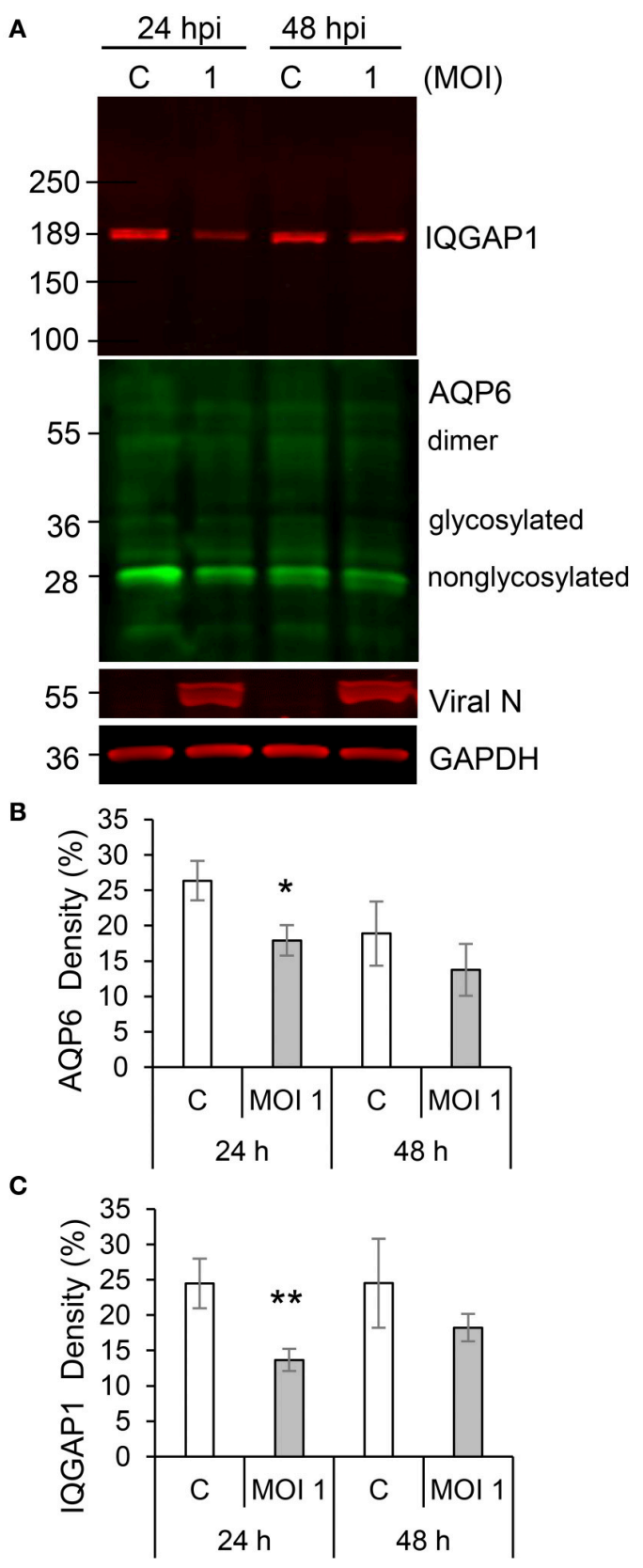

FIGURE 5 | Hazara virus affects the level of IQGAP1 and AQP6 in epithelial cells. (A) Cells were untreated controls (c) or infected with Hazara virus at $\mathrm{MOI}$ 1 for $1 \mathrm{~h}$ and maintained for 24 and $48 \mathrm{hpi}$. Immunoblots for IQGAP1, AQP6, viral $\mathrm{N}$, and $\mathrm{GAPDH}$. The blots are from one representative out of four independent experiments: (B,C) Quantification of blots. AQP6 and IQGAP1 levels normalized to the GAPDH control are indicated as percent (\%). Values are the mean \pm SE based on four independent experiments performed on separate days from different cell passages $(n=4)$. Significant differences were analyzed by Student's $t$-test and are indicated with * or ${ }^{* *}$ when $P<0.05$ or $P<0.01$ compared to control.

decreased repair of the wounds, e.g., after longer presence of the virus (Figures 4, 5) and associated disturbances in the cellular proteome. During virus infection, a plethora of factors released

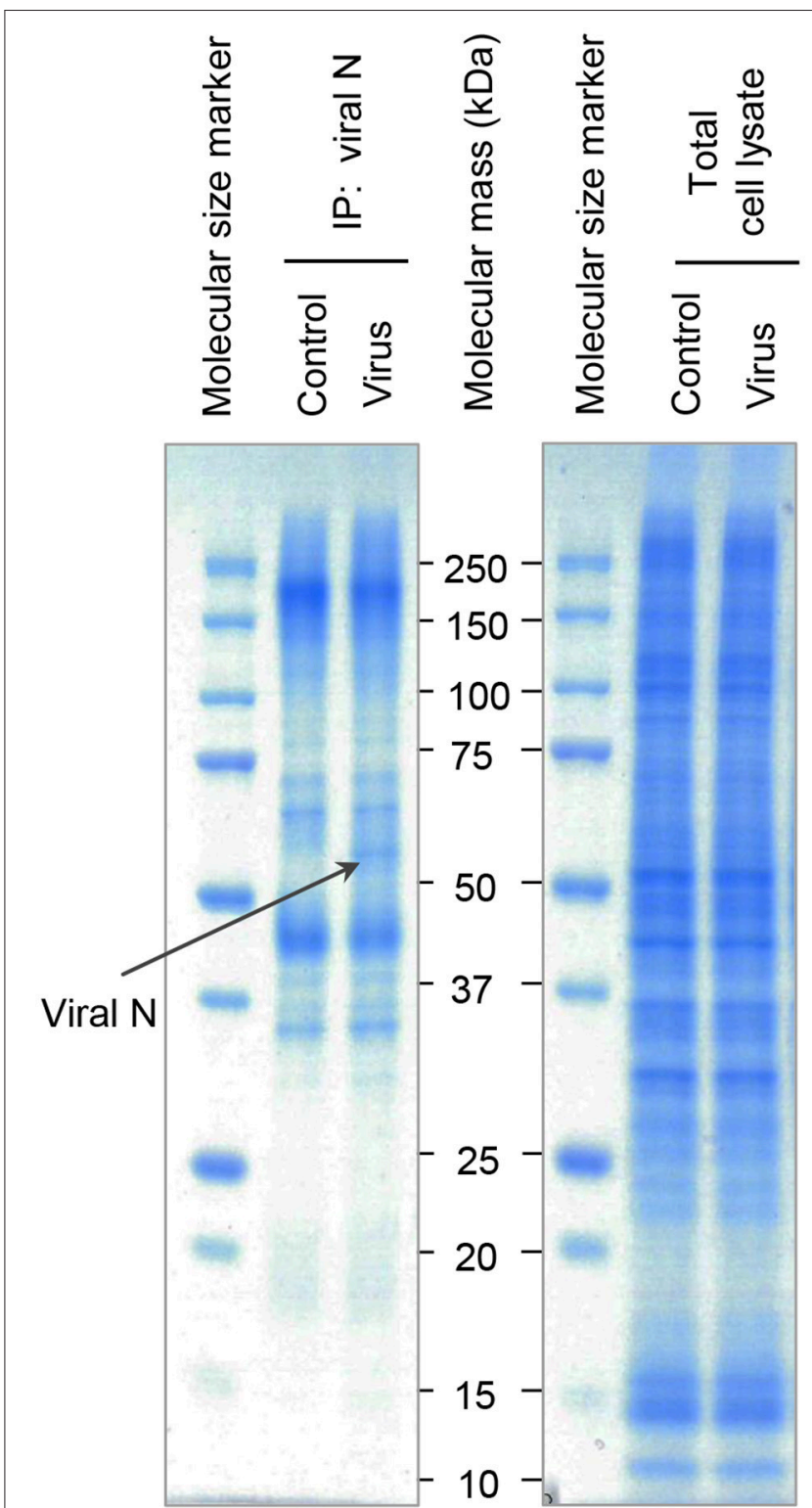

FIGURE 6 | SDS-PAGE analysis of N-associated immune complexes of epithelial cells. Cells were untreated (Control) or infected with Hazara virus at $\mathrm{MOI} 1$ for $1 \mathrm{~h}$ and maintained for $24 \mathrm{hpi}$ (Virus). Total cell lysates were immunoprecipitated using the antibodies against CCHFV/Hazara (viral N). $\mathrm{N}$-enriched immune complexes (Left Panel) and total cell lysates as a control (Right Panel) were analyzed by SDS-PAGE and stained with Coomassie Blue. Bands were excised and N-binding cellular proteins identified by in-gel digestion and LC-MS/MS analysis as shown in Table 1. The band indicated with a black arrow represent viral N. Displayed are representative gels from one of four independent experiments.

from the cells may also have some impact on epithelial repair and barrier function (Bente et al., 2013).

Successful wound closure requires extensive remodeling of the cytoskeleton, which is controlled by a plethora molecules (Yang et al., 2009; Ivanov et al., 2010; Friedl and Mayor, 2017), and among them, scaffold IQGAP1 (Karlsson et al., 2012; Hedman 
TABLE 1 | Alterations in the human cellular interactome of the Hazara virus $\mathrm{N}$ compared to the control non-infected cells.

\begin{tabular}{|c|c|c|c|c|c|c|c|c|c|c|c|c|}
\hline Identified proteins & $\begin{array}{l}\text { Gene } \\
\text { (Uniprot } \\
\text { accession } \\
\text { number) }\end{array}$ & $\begin{array}{l}\text { Protein } \\
\text { (Uniprot } \\
\text { accession } \\
\text { number) }\end{array}$ & MW kDa & $P$-value & $\begin{array}{l}\text { Fold change, } \\
\text { (infected/control) }\end{array}$ & \multicolumn{7}{|c|}{ Functional groups of human proteins \# } \\
\hline \multicolumn{13}{|c|}{ PROTEINS DETECTED IN N-ENRICHED IMMUNE COMPLEXES ONLY IN VIRUS-INFECTED CELLS } \\
\hline Calnexin & CANX & P27824 & 72 & 0.0001 & $\begin{array}{l}\text { Detected only in } \\
\text { virus-infected cells }\end{array}$ & $\mathbf{X}$ & & $\mathbf{X}$ & $\mathrm{X}$ & $\mathbf{X}$ & $\mathbf{x}$ & $\mathbf{x}$ \\
\hline Probable serine carboxypeptidase CPVL & CPVL & Q9H3G5 & 54 & 0.024 & & & $\mathrm{x}$ & & & & & $\mathbf{x}$ \\
\hline 14-3-3 protein beta/alpha & YWHAB & P31946 & 28 & 0.025 & & $\mathbf{X}$ & & $\mathbf{X}$ & $x$ & $\mathbf{X}$ & & $\mathbf{x}$ \\
\hline Stress-induced-phosphoprotein 1 & STIP1 & P31948 & 68 & 0.025 & & $\mathbf{X}$ & & $\mathbf{X}$ & & & $x$ & $\mathbf{x}$ \\
\hline LIM and SH3 domain protein 1 & LASP1 & Q14847 & 30 & 0.037 & & & & & & $\mathbf{X}$ & & $\mathbf{x}$ \\
\hline Dihydropyrimidinase-related protein 2 & DPYSL2 & Q16555 & 62 & 0.045 & & & & & & $\mathbf{X}$ & & $\mathbf{x}$ \\
\hline Ryanodine receptor 3 & RYR3 & Q15413 & 552 & 0.046 & & & & & $x$ & $X$ & & \\
\hline $\begin{array}{l}\text { Low-density lipoprotein receptor-related } \\
\text { protein 1B }\end{array}$ & LRP1B & Q9NZR2 & 515 & 0.054 & & & & $X$ & & & & \\
\hline Peroxiredoxin-1 & PRDX1 & Q06830 & 19 & 0.058 & & $\mathbf{X}$ & $\mathrm{x}$ & $\mathbf{X}$ & & $\mathbf{X}$ & & $\mathbf{x}$ \\
\hline Protein disulfide isomerase & TXNDC5 & Q86UY0 & 40 & 0.083 & & & & $\mathbf{x}$ & & & $\mathbf{x}$ & $\mathbf{x}$ \\
\hline
\end{tabular}

BLOC1S5-TXNDC5

\section{PROTEINS DISPLAYED INCREASED BINDING TO N-ENRICHED IMMUNE COMPLEXES UPON VIRUS INFECTION}

Hsc70-interacting protein

Fructose-bisphosphate aldolase A

F-box only protein 2

$60 \mathrm{kDa}$ heat shock protein, mitochondrial, HSP60

Annexin A2

Malate dehydrogenase, cytoplasmic

Phosphoglycerate kinase 1

Villin-1

Protein disulfide-isomerase A4

Regulation of nuclear pre-mRNA

domain-containing protein $1 \mathrm{~B}$

Alpha-enolase

Elongation factor 1-delta

Transaldolase

Polyadenylate-binding protein

Nucleolysin TIAR

Non-POU domain-containing

octamer-binding protein

Heterogeneous nuclear ribonucleoprotein $Q$

Stress-70 protein, mitochondrial, HSP70

ATP-dependent RNA

Histone $\mathrm{H} 1.5$

28 S ribosomal protein S29, mitochondrial

RNA-binding protein EWS

Histone-arginine methyltransferase CARM1

Guanine nucleotide-binding protein subunit

beta-2-like 1, Receptor of activated protein

C kinase 1

Heterogeneous nuclear ribonucleoprotein $\mathrm{L}$

$\begin{array}{lllll}\text { ST13 } & \text { H7C3I1 } & 16 & 0.0002 & 14 \\ \text { ALDOA } & \text { P04075 } & 45 & 0.013 & 19 \\ \text { FBXO2 } & \text { Q9UK22 } & 33 & 0.028 & 2.8 \\ \text { HSPD1 } & \text { P10809 } & 61 & 0.029 & 2.2 \\ & & & & \\ \text { ANXA2 } & \text { P07355 } & 40 & 0.029 & 3.6 \\ \text { MDH1 } & \text { P40925 } & 39 & 0.051 & 3.9 \\ \text { PGK1 } & \text { P00558 } & 45 & 0.064 & 2.6 \\ \text { VIL1 } & \text { P09327 } & 93 & 0.066 & 2.4 \\ \text { PDIA4 } & \text { P13667 } & 73 & 0.086 & 3.7 \\ \text { RPRD1B } & \text { Q9NQG5 } & 37 & 0.094 & 5.6 \\ & & & & \\ \text { ENO1 } & \text { P06733 } & 47 & 0.095 & 2.1 \\ \text { EEF1D } & \text { P29692 } & 69 & 0.097 & 2.4 \\ \text { TALDO1 } & \text { P37837 } & 38 & 0.1 & 3.1\end{array}$

$\mathrm{x} \quad \mathrm{x}$

$\begin{array}{ll} & X \quad X \\ X & X\end{array}$

3.9

.6

3.7

6

$\begin{array}{llll}x & x & x \\ x & x & x\end{array}$

$\mathbf{X}$

$\begin{array}{rrrr} & x & x & \\ x & & x & \\ x & & x & \\ x & & x & \\ x & x & x & x \\ & & x & \end{array}$

X $\mathrm{X}$

$x \quad x \quad x$

X $\mathrm{X}$

X

$\mathrm{X}$

$3.1 \quad X$

\section{$\mathrm{X}$}

$\mathrm{X}$

$\mathbf{x}$

$\begin{array}{lllll}\text { TIAL1 } & \text { Q01085 } & 43 & 0.018 & 0.7\end{array}$

$\begin{array}{lllll}\text { NONO } & \text { Q15233 } & 54 & 0.035 & 0.4\end{array}$

$\mathbf{X}$

$\mathbf{X}$

$\mathbf{X}$

$\begin{array}{lllll}\text { SYNCRIP } & 060506 & 70 & 0.052 & 0.5\end{array}$

$\begin{array}{lllll}\text { HSPA9 } & \text { P38646 } & 74 & 0.052 & 0.7\end{array}$

$\begin{array}{lllll}\text { DDX3X } & 000571 & 71 & 0.053 & 0.5\end{array}$

$\begin{array}{llll}\text { HIST1H1B P16401 } 23 & 0.065 & 0.3\end{array}$

$\begin{array}{lllll}\text { DAP3 } & \text { P51398 } & 46 & 0.077 & 0.4\end{array}$

$\begin{array}{lllll}\text { EWSR1 } & \text { Q01844 } & 63 & 0.077 & 0.8\end{array}$

$\begin{array}{lllll}\text { CARM1 Q86X55 } & 63 & 0.083 & 0.6\end{array}$

$\begin{array}{lllll}\text { GNB2L1 } & \text { P63244 } & 35 & 0.089 & 0.4\end{array}$

HNRNL P14866 64

$0.091 \quad 0.7$

X

$\mathbf{X}$

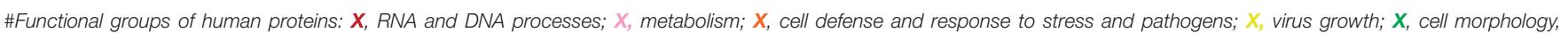

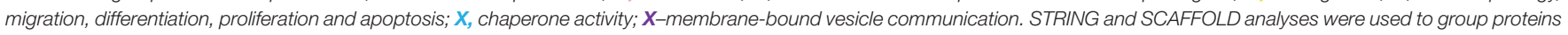
into functional classes. Data are from 4 independent experiments. 


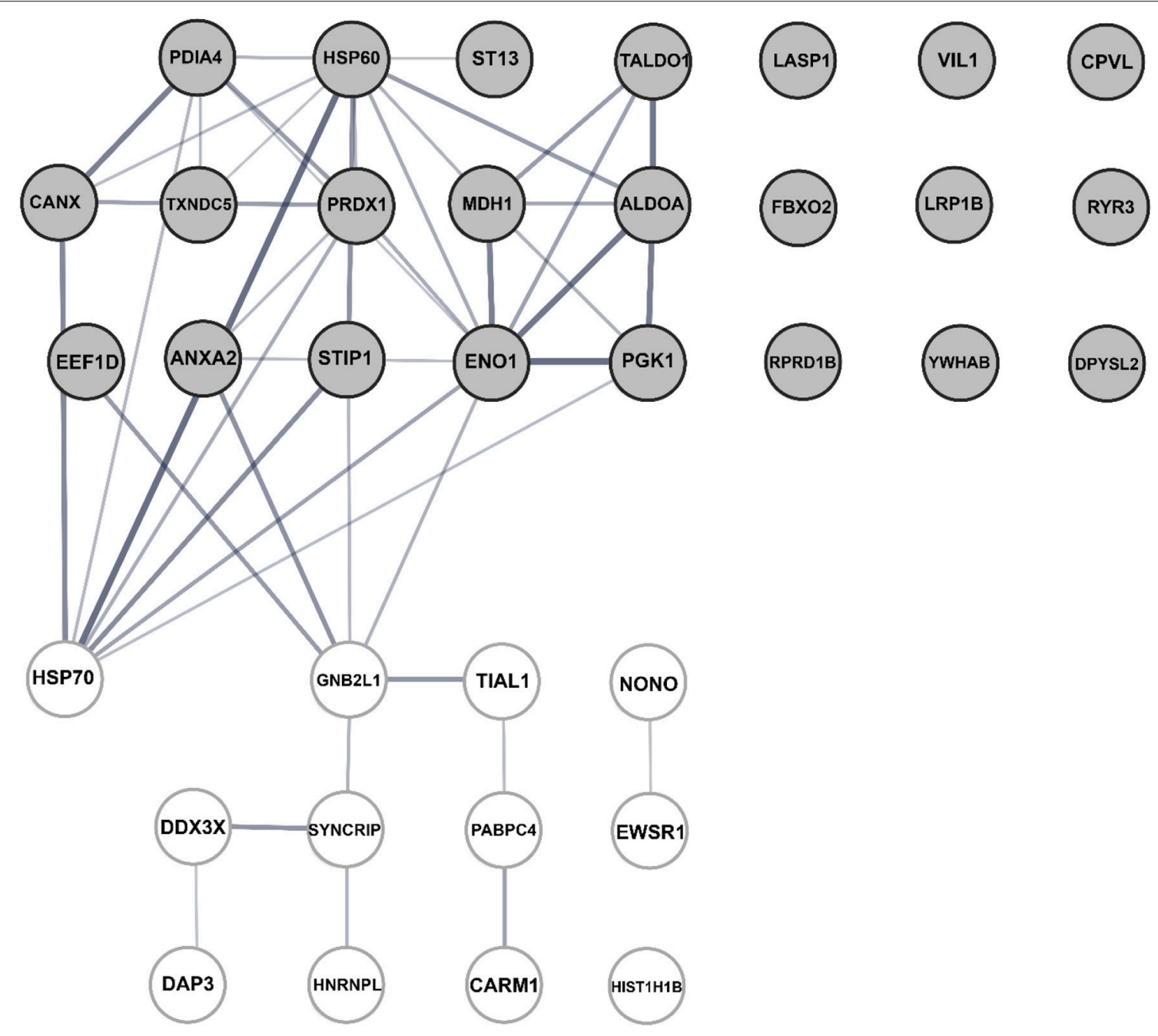

FIGURE 7 | Bioinformatic assessment of the human cellular interactome of the Hazara virus N shown in Table 1. Network nodes represent human proteins pooled into two units. The upper unit with gray nodes represent 23 proteins suggested as potential $\mathrm{N}$ partners upon Hazara infection. These include $10 \mathrm{~N}$-associated proteins detected only in virus-infected cells and 13 proteins displayed at least 2-fold increased binding to $\mathrm{N}$-enriched immune complexes upon viral infection in comparison to non-infected control (Table 1). The lower unit with white nodes represent 12 proteins displayed decreased binding to $\mathrm{N}$-enriched immune complexes upon viral infection in comparison to non-infected control (Table 1). Edges represent protein-protein interactions where line thickness indicates the strength of data support. The assessment is based on STRING analysis shown in Figure $\mathbf{S 1}$ and LC-MS/MS analysis and quantification of alterations in cellular proteome and interactome of N shown in Table 1.

et al., 2015) and AQP (Loitto et al., 2009; Karlsson et al., 2013), playing a pivotal role in regulation of cell migration, cytoskeleton dynamics, water homeostasis and vesicle communication. We therefore further focused our investigations on the mechanisms whereby Hazara virus could have on epithelial cell migration. Using confocal imaging and immunoblotting, we observed that the F-actin was disrupted and the expression and distribution of IQGAP1 and AQP6 were reduced upon Hazara infection (Figures 4, 5). This may allow virus to maintain long-term infections in the host and perturb the repair of epithelia by interfering with cytoskeletal structure and water homeostasis.

Our findings corroborate several reports on how microbial pathogens may manipulate signaling pathways of the host cells, particularly cytoskeletal dynamics, to help them invade, replicate and maintain infections (Chazal and Gerlier, 2003; Sewald et al., 2016). Thus, IQGAP1 regulates egress of Ebola virus (Lu et al., 2013), invasion and replication of Moloney leukemia virus (Leung et al., 2006) and virulence of swine fever virus (Gladue et al., 2011). Moreover, CCHFV did perturb strongly the microtubule and actin filaments during entry, growth and release to the surroundings (Andersson et al., 2004; Simon et al., 2009). Additionally, other studies have proposed a new role for AQP as important proteins during viral infections and inflammation. Thus, the levels of AQP1 and 5 were low during pulmonary, adenoviral infection (Towne et al., 2000). AQP4 was decreased in the acute phase of a Herpes simplex virus infection, but increased in the long-term of disease (Martinez Torres et al., 2007). An infection of human cells with CCHFV strain IbAR 
10200 downregulated the AQP6 mRNA expression (Molinas et al., 2016). Furthermore, with Hazara virus as a model, we have recently provided an evidence for a protective role of AQP6 against virus infection (Molinas et al., 2016).

To further elucidate details on how the virus affects the cells, we aimed to identify potential partners of Hazara virus $\mathrm{N}$ in the epithelial cellular proteome upon infection. To do this, we employed immunoprecipitation, SDS-PAGE (Figure 6), LC-MS/MS (Table 1) and bioinformatics analyses (Figure 7). Upon virus infection, 36 out of around 500 cellular proteins were typically identified in $\mathrm{N}$-enriched immune complexes and exhibited significant difference in in connectivity to viral $\mathrm{N}$ (Table 1). Among them, 10 proteins were detected only in virus-infected cells, and further 13 and 12 proteins displayed either increased or decreased binding to $\mathrm{N}$-enriched immune complexes, respectively, upon viral infection in comparison to non-infected control (Table 1).

We identified proteins with chaperone activity (Figure 7, Table 1, noted in blue). They can interact with viral proteins, including $\mathrm{N}$, and favor viral replication and block actin binding (Mirazimi et al., 1998; Horna-Terron et al., 2014; Abbas et al., 2015; Khachatoorian and French, 2016). Both Hsp60 and Hsp70, may also directly bind virus RNA (Nanda et al., 2004) and thereby control viral infection (Lahaye et al., 2012). Consistent with this scenario, Hsp70 association with Nairovirus $\mathrm{N}$ was required for viral replication (Surtees et al., 2016).

We also recognized the ribosomal receptor of activated protein $\mathrm{C}$ kinase 1 and cytoplasmic heterogeneous nuclear ribonucleoprotein Q (Figure 7), earlier being implicated in viral replication and release (Liu et al., 2009; Demirov et al., 2012).

A cluster of the proteins is involved in RNA and DNA processes (Table 1, noted in red). Viral $\mathrm{N}$ has several RNA binding domains that facilitate ribonucleoprotein formation in mature virions. This should also give a basis for molecular mimicry allowing $\mathrm{N}$ to associate with host cellular RNA-binding proteins, to be transported to the nuclear region and to utilize host protein machinery for virus production (Rowland and Yoo, 2003; Wulan et al., 2015).

A group of N-binding proteins orchestrates membrane-bound vesicle communication (Table 1, shown in violet). Extracellular membrane vesicles (EMV) are membrane-bound structures shedding from cells into the environment and playing a role in cell-cell communication through protein, lipid and nucleic acid transfer. Both pathogenic bacteria and viruses can hijack

\section{Epithelium}

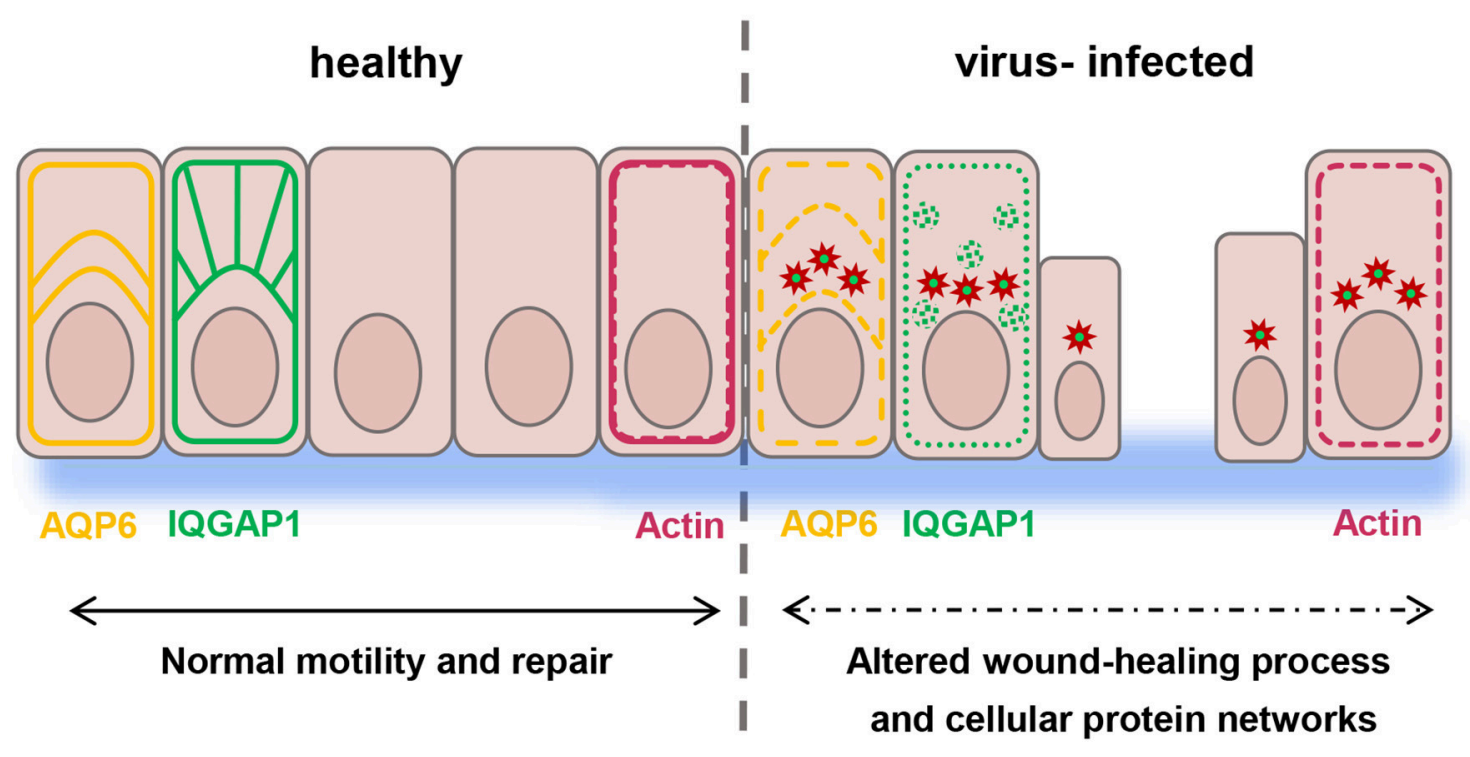

Function

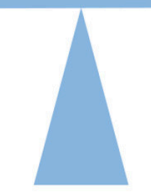

Dysfunction

FIGURE 8 | Schematic model. Epithelial wound healing process and cellular protein networks (shown as arrows) in human epithelial cells (in pink) are perturbed by Hazara virus infection. This is paralleled by disruption in the organization of actin cytoskeleton (in red) and reduction of the expression and distribution of IQGAP1 (in green) and AQP6 (in yellow), events that influence homeostasis in the intestine and along blood vessel walls and integrity of an individual. 
host EMV and thereby enhance their pathogenicity (Schwab et al., 2015; Turkina et al., 2015). Coxsackie virus disseminates within host EMV upon stem cell migration and differentiation and thereby spreads to new cells (Robinson et al., 2014). Severe fever with thrombocytopenia syndrome virus (SFTS), which is a new Bunyaviridae family member, incorporates itself into EMV and use them as a delivery system for its own spreading (Silvas et al., 2016). We suggest that Hazara virus may also take the advantage of host EMV to promote spreading through cell-tocell contacts and thereby the establishment and progression of infection.

A large group of proteins is required for regulation of cell morphology, migration, differentiation, proliferation and apoptosis (Table 1, shown in green). Indeed, virus can target and manipulate host cytoskeleton dynamics and organization which may ensure virus replication and spread (Sewald et al., 2016). Dendritic cells (Cunningham et al., 2010), monocytes (DaleyBauer et al., 2014), and T-cells (Murooka et al., 2012) can be hijacked by varicella zoster virus, cytomegalovirus and HIV, and thereby used as migratory vehicles for viral dissemination.

In many RNA viruses, $\mathrm{N}$ is known not only as a structural but also as a functionally important protein. Thus, $\mathrm{N}$ binds to the viral RNA genome and forms ribonucleoproteins in mature virions, which is seen in severe acute respiratory syndrome coronavirus (Huang et al., 2004), infectious bronchitis virus (Spencer and Hiscox, 2006), and CCHFV (Morikawa et al., 2007). Viral N interferes with immune system to enhance virus virulence, arrest host cell cycle and inactivate chaperons (Emmott et al., 2013; McBride et al., 2014). By such a broader interaction with host cell proteins, directly or in-directly, $\mathrm{N}$ may manipulate host cytoskeleton dynamics and other distinct cellular processes that potentially affect the virus life cycle.

In summary, our study demonstrates that Hazara virus can modulate migration of human epithelial cells, disrupt their actin cytoskeleton organization, cellular distribution and reduce the expression of IQGAP1 and AQP6, which help regulate cytoskeleton dynamics and water homeostasis. Moreover, upon infection, Hazara virus structural $\mathrm{N}$ is engaged with an array of cellular protein interactions (Figure 8). Our interactome data provide a foundation for future work on mechanistic insights into biology of an infection. Taken together, these events interfere with many cellular processes that potentially facilitate the virus life cycle, but they are also deleterious to cell renewal and wound healing at epithelial and endothelial linings being so essential to organism homeostasis under normal physiological situation and after bacterial or virus injury and infection.

\section{AUTHOR CONTRIBUTIONS}

EV conceived and designed the experiments; EV, MT, and AM performed the experiments; EV, MT, AM, and K-EM contributed to the data analysis and interpretation of the results; AliM contributed materials; EV drafted the manuscript; EV, MT, AM, and K-EM edited the manuscript; All authors approved the final version of the manuscript.

\section{FUNDING}

This research work was supported by grants from the Swedish Research Council, the European Science foundation (TraPPs Euromembrane project), Magnus Bergvall Foundation, Olle Engkvist Foundation, the Faculty of Medicine and Health Sciences, Linköping University and Infect-ERA Second Call (Swedish Research Council, 214-7495).

\section{SUPPLEMENTARY MATERIAL}

The Supplementary Material for this article can be found online at: https://www.frontiersin.org/articles/10.3389/fcell.2017. 00098/full\#supplementary-material

Figure S1 | Visualization of Hazara virus, F-actin, IQGAP1 and AQP6 in epithelial cells. Cells were untreated (Control) or infected with Hazara virus at $\mathrm{MOI} 1$ for $1 \mathrm{~h}$ and maintained for $24 \mathrm{hpi}$. Samples were then fixed and stained for: (A) viral $\mathrm{N}$ (red) and F-actin (green); (B) viral N (red) and IQGAP1 (green); (C) viral N (green) and AQP6 (red); nuclei were labeled with DAPI (blue). Samples were analyzed by confocal microscopy. The data is from one representative of three independent experiments. Image size is $67.6 \times 67.6 \mu \mathrm{m}$ and pixel size is $0.13 \mu \mathrm{m}$. Quantification of immunofluorescence intensity profiles for F-actin (D), IQGAP1 (E) and AQP6 (F) measured across the cell monolayers as indicated by blue arrows (control) and yellow arrows (virus-infected cells, MOI 1, 24 hpi). The length of arrows reflects the distance of $60 \mu \mathrm{m}$.

Figure S2 | Additional quantification of F-actin fluorescence intensity profiles. The set of experiments were performed and quantification of fluorescence intensity profiles were done as in Figure 4A and Figures S1A,D. Non-infected cells are indicated by blue arrows (control) and virus-infected cells are coded by yellow arrows (MOI 1, $24 \mathrm{hpi}$ ). The length of arrows reflects the distance of $60 \mu \mathrm{m}$. Shown are F-actin intensity profiles measured across the cell monolayers from 6 representative cell regions and three independent experiments performed on separate days from different cell passages.

Figure S3 | Additional quantification of IQGAP1 fluorescence intensity profiles. The set of experiments were performed and quantification of fluorescence intensity profiles were done as in Figure 4B and Figures S1B,E. Non-infected cells are indicated by blue arrows (control) and virus-infected cells are coded by yellow arrows (MOI 1, $24 \mathrm{hpi}$ ). The length of arrows reflects the distance of $60 \mu \mathrm{m}$. Shown are IQGAP1 intensity profiles measured across the cell monolayers from 6 representative cell regions and three independent experiments performed on separate days from different cell passages.

Figure S4 | Additional quantification of AQP6 fluorescence intensity profiles. The set of experiments were performed and quantification of fluorescence intensity profiles were done as in Figure $\mathbf{4 C}$ and Figures S1C,F. Non-infected cells are indicated by blue arrows (control) and virus-infected cells are coded by yellow arrows (MOI 1, $24 \mathrm{hpi}$ ). The length of arrows reflects the distance of $60 \mu \mathrm{m}$. Shown are AQP6 intensity profiles measured across the cell monolayers from 6 representative cell regions and three independent experiments performed on separate days from different cell passages.

Figure S5 | Bioinformatic STRING analysis of the human cellular interactome of the Hazara virus $\mathrm{N}$ shown in Table 1. Colored network nodes represent query proteins. Edges represent protein-protein interactions and include different type of actions depicted by the colored lines. For known interactions: pink, experimentally determined; turquoise, from curated databases. For predicted interactions: green, gene neighborhood; blue, gene co-occurrence. For others interactions: olive green, literature mining; black, co-expression; purple, protein homology.

Figure S6 | Additional STRING bioinformatic analysis of the human cellular interactome of the Hazara virus N shown in Table 1. Network nodes represent proteins. Red nodes, query proteins and first shell of interaction for those involved in (A) RNA and DNA processes and those associated to (B) membrane bond vesicles. White nodes, second shell of interaction. Edges represent protein-protein interactions and include different type of actions depicted by the colored lines as in Figure S1. 


\section{REFERENCES}

Abbas, W., Kumar, A., and Herbein, G. (2015). The eEF1A proteins: at the crossroads of oncogenesis, apoptosis, and viral infections. Front. Oncol. 5:75. doi: $10.3389 /$ fonc. 2015.00075

Andersson, I., Simon, M., Lundkvist, A., Nilsson, M., Holmstrom, A., Elgh, F., et al. (2004). Role of actin filaments in targeting of Crimean Congo hemorrhagic fever virus nucleocapsid protein to perinuclear regions of mammalian cells. $J$. Med. Virol. 72, 83-93. doi: 10.1002/jmv.10560

Beitz, E., Liu, K., Ikeda, M., Guggino, W. B., Agre, P., and Yasui, M. (2006). Determinants of AQP6 trafficking to intracellular sites versus the plasma membrane in transfected mammalian cells. Biol. Cell 98, 101-109. doi: $10.1042 /$ BC20050025

Benga, G. (2012). On the definition, nomenclature and classification of water channel proteins (aquaporins and relatives). Mol. Aspects Med. 33, 514-517. doi: 10.1016/j.mam.2012.04.003

Bente, D. A., Forrester, N. L., Watts, D. M., McAuley, A. J., Whitehouse, C. A., and Bray, M. (2013). Crimean-Congo hemorrhagic fever: history, epidemiology, pathogenesis, clinical syndrome and genetic diversity. Antiviral Res. 100, 159-189. doi: 10.1016/j.antiviral.2013.07.006

Chazal, N., and Gerlier, D. (2003). Virus entry, assembly, budding, and membrane rafts. Microbiol. Mol. Biol. Rev. 67, 226-237. doi: 10.1128/MMBR.67.2.226-237.2003

Cohen, S., Au, S., and Pante, N. (2011). How viruses access the nucleus. Biochim. Biophys. Acta 1813, 1634-1645. doi: 10.1016/j.bbamcr.2010.12.009

Condeelis, J., and Pollard, J. W. (2006). Macrophages: obligate partners for tumor cell migration, invasion, and metastasis. Cell 124, 263-266. doi: 10.1016/j.cell.2006.01.007

Connolly-Andersen, A. M., Magnusson, K. E., and Mirazimi, A. (2007). Basolateral entry and release of Crimean-Congo hemorrhagic fever virus in polarized MDCK-1 cells. J. Virol. 81, 2158-2164. doi: 10.1128/JVI.02070-06

Cunningham, A. L., Donaghy, H., Harman, A. N., Kim, M., and Turville, S. G. (2010). Manipulation of dendritic cell function by viruses. Curr. Opin. Microbiol. 13, 524-529. doi: 10.1016/j.mib.2010.06.002

Daley-Bauer, L. P., Roback, L. J., Wynn, G. M., and Mocarski, E. S. (2014). Cytomegalovirus hijacks CX3CR1(hi) patrolling monocytes as immuneprivileged vehicles for dissemination in mice. Cell Host Microbe 15, 351-362. doi: 10.1016/j.chom.2014.02.002

Demirov, D., Gabriel, G., Schneider, C., Hohenberg, H., and Ludwig, S. (2012). Interaction of influenza A virus matrix protein with RACK1 is required for virus release. Cell. Microbiol. 14, 774-789. doi: 10.1111/j.1462-5822.2012.01759.x

Dowall, S. D., Buttigieg, K. R., Findlay-Wilson, S. J., Rayner, E., Pearson, G., Miloszewska, A., et al. (2015). A Crimean-Congo Haemorrhagic Fever (CCHF) viral vaccine expressing nucleoprotein is immunogenic but fails to confer protection against lethal disease. Hum. Vaccin. Immunother. 12, 519-527. doi: $10.1080 / 21645515.2015$

Dowall, S. D., Findlay-Wilson, S., Rayner, E., Pearson, G., Pickersgill, J., Rule, A., et al. (2012a). Hazara virus infection is lethal for adult type I interferon receptor-knockout mice and may act as a surrogate for infection with the human-pathogenic Crimean-Congo hemorrhagic fever virus. J. Gen. Virol. 93, 560-564. doi: 10.1099/vir.0.038455-0

Dowall, S. D., Richards, K. S., Graham, V. A., Chamberlain, J., and Hewson, R. (2012b). Development of an indirect ELISA method for the parallel measurement of $\operatorname{IgG}$ and IgM antibodies against CrimeanCongo haemorrhagic fever (CCHF) virus using recombinant nucleoprotein as antigen. J. Virol. Methods 179, 335-341. doi: 10.1016/j.jviromet.2011. 11.020

Emmott, E., Munday, D., Bickerton, E., Britton, P., Rodgers, M. A., Whitehouse, A., et al. (2013). The cellular interactome of the coronavirus infectious bronchitis virus nucleocapsid protein and functional implications for virus biology. $J$. Virol. 87, 9486-9500. doi: 10.1128/JVI.00321-13

Ergonul, O. (2006). Crimean-Congo haemorrhagic fever. Lancet Infect. Dis. 6, 203-214. doi: 10.1016/S1473-3099(06)70435-2

Ergonul, O. (2012). Crimean-Congo hemorrhagic fever virus: new outbreaks, new discoveries. Curr. Opin. Virol. 2, 215-220. doi: 10.1016/j.coviro.2012.03.001

Franceschini, A., Szklarczyk, D., Frankild, S., Kuhn, M., Simonovic, M., Roth, A., et al. (2013). STRING v9.1: protein-protein interaction networks, with increased coverage and integration. Nucleic Acids Res. 41, D808-D815. doi: 10.1093/nar/gks1094

Friedl, P., and Mayor, R. (2017). Tuning collective cell migration by cellcell junction regulation. Cold Spring Harb. Perspect. Biol. 9:a029199. doi: 10.1101/cshperspect.a029199

Gladue, D. P., Holinka, L. G., Fernandez-Sainz, I. J., Prarat, M. V., O’Donnell, V., Vepkhvadze, N. G., et al. (2011). Interaction between Core protein of classical swine fever virus with cellular IQGAP1 protein appears essential for virulence in swine. Virology 412, 68-74. doi: 10.1016/j.virol.2010.12.060

Hedman, A. C., Smith, J. M., and Sacks, D. B. (2015). The biology of IQGAP proteins: beyond the cytoskeleton. EMBO Rep. 16, 427-446. doi: 10.15252/embr.201439834

Holm, A., Magnusson, K. E., and Vikstrom, E. (2016). Pseudomonas aeruginosa $\mathrm{N}$-3-oxo-dodecanoyl-homoserine lactone elicits changes in cell volume, morphology, and AQP9 characteristics in macrophages. Front. Cell. Infect. Microbiol. 6:32. doi: 10.3389/fcimb.2016.00032

Honig, J. E., Osborne, J. C., and Nichol, S. T. (2004). The high genetic variation of viruses of the genus Nairovirus reflects the diversity of their predominant tick hosts. Virology 318, 10-16. doi: 10.1016/j.virol.2003.09.021

Horna-Terron, E., Pradilla-Dieste, A. C., Sanchez-de-Diego, and Osada, J. (2014). TXNDC5, a newly discovered disulfide isomerase with a key role in cell physiology and pathology. Int. J. Mol. Sci. 15, 23501-23518. doi: $10.3390 /$ ijms 151223501

Huang, Q., Yu, L., Petros, A. M., Gunasekera, A., Liu, Z., Xu, N., et al. (2004). Structure of the N-terminal RNA-binding domain of the SARS CoV nucleocapsid protein. Biochemistry 43, 6059-6063. doi: 10.1021/bi036155b

Ivanov, A. I., Parkos, C. A., and Nusrat, A. (2010). Cytoskeletal regulation of epithelial barrier function during inflammation. Am. J. Pathol. 177, 512-524. doi: 10.2353/ajpath.2010.100168

Karlsson, T., Lagerholm, B. C., Vikstrom, E., Loitto, V. M., and Magnusson, K. E. (2013). Water fluxes through aquaporin-9 prime epithelial cells for rapid wound healing. Biochem. Biophys. Res. Commun. 430, 993-998. doi: $10.1016 /$ j.bbrc.2012.11.125

Karlsson, T., Turkina, M. V., Yakymenko, O., Magnusson, K. E., and Vikstrom, E. (2012). The Pseudomonas aeruginosa $\mathrm{N}$-acylhomoserine lactone quorum sensing molecules target IQGAP1 and modulate epithelial cell migration. PLoS Pathog. 8:e1002953. doi: 10.1371/journal.ppat.1002953

Khachatoorian, R., and French, S. W. (2016). Chaperones in hepatitis C virus infection. World J. Hepatol. 8, 9-35. doi: 10.4254/wjh.v8.i1.9

Lahaye, X., Vidy, A., Fouquet, B., and Blondel, D. (2012). Hsp70 protein positively regulates rabies virus infection. J. Virol. 86, 4743-4751. doi: 10.1128/JVI.06501-11

Leung, J., Yueh, A., Appah, F. S. Jr., Yuan, B., de los Santos, K., and Goff, S. P. (2006). Interaction of Moloney murine leukemia virus matrix protein with IQGAP. EMBO J. 25, 2155-2166. doi: 10.1038/sj.emboj.7601097

Liu, H. M., Aizaki, H., Choi, K. S., Machida, K., Ou, J. J., and Lai, M. M. (2009). SYNCRIP (synaptotagmin-binding, cytoplasmic RNA-interacting protein) is a host factor involved in hepatitis C virus RNA replication. Virology 386, 249-256. doi: 10.1016/j.virol.2009.01.018

Loitto, V. M., Karlsson, T., and Magnusson, K. E. (2009). Water flux in cell motility: expanding the mechanisms of membrane protrusion. Cell Motil. Cytoskeleton 66, 237-247. doi: $10.1002 / \mathrm{cm} .20357$

Lu, J., Qu, Y., Liu, Y., Jambusaria, R., Han, Z., Ruthel, G., et al. (2013). Host IQGAP1 and Ebola virus VP40 interactions facilitate virus-like particle egress. J. Virol. 87, 7777-7780. doi: 10.1128/JVI.00470-13

Martinez Torres, F. J., Volcker, D., Dorner, N., Lenhard, T., Nielsen, S., Haas, J., et al. (2007). Aquaporin 4 regulation during acute and long-term experimental Herpes simplex virus encephalitis. J. Neurovirol. 13, 38-46. doi: $10.1080 / 13550280601145340$

McBride, R., van Zyl, M., and Fielding, B. C. (2014). The coronavirus nucleocapsid is a multifunctional protein. Viruses 6, 2991-3018. doi: 10.3390/v6082991

Mirazimi, A., Nilsson, M., and Svensson, L. (1998). The molecular chaperone calnexin interacts with the NSP4 enterotoxin of rotavirus in vivo and in vitro. J. Virol. 72, 8705-8709.

Molinas, A., Mirazimi, A., Holm, A., Loitto, V. M., Magnusson, K. E., and Vikstrom, E. (2016). Protective role of host aquaporin 6 against Hazara virus, a model for Crimean-Congo hemorrhagic fever virus infection. FEMS Microbiol. Lett. 363:fnw058. doi: 10.1093/femsle/fnw058 
Morikawa, S., Saijo, M., and Kurane, I. (2007). Recent progress in molecular biology of Crimean-Congo hemorrhagic fever. Comp. Immunol. Microbiol. Infect. Dis. 30, 375-389. doi: 10.1016/j.cimid.2007.07.001

Murooka, T. T., Deruaz, M., Marangoni, F., Vrbanac, V. D., Seung, E., von Andrian, U. H., et al. (2012). HIV-infected T cells are migratory vehicles for viral dissemination. Nature 490, 283-287. doi: 10.1038/nature11398

Nanda, S. K., Johnson, R. F., Liu, Q., and Leibowitz, J. L. (2004). Mitochondrial HSP70, HSP40, and HSP60 bind to the $3^{\prime}$ untranslated region of the Murine hepatitis virus genome. Arch. Virol. 149, 93-111. doi: 10.1007/s00705-003-0196-4

Nesvizhskii, A. I., Keller, A., Kolker, E., and Aebersold, R. (2003). A statistical model for identifying proteins by tandem mass spectrometry. Anal. Chem. 75, 4646-4658. doi: 10.1021/ac0341261

Nozaki, K., Ishii, D., and Ishibashi, K. (2008). Intracellular aquaporins: clues for intracellular water transport? Pflugers Arch. 456, 701-707. doi: 10.1007/s00424-007-0373-5

Robinson, S. M., Tsueng, G., Sin, J., Mangale, V., Rahawi, S., McIntyre, L. L., et al. (2014). Coxsackievirus B exits the host cell in shed microvesicles displaying autophagosomal markers. PLoS Pathog. 10:e1004045. doi: 10.1371/journal.ppat.1004045

Rodrigues, S. F., and Granger, D. N. (2015). Blood cells and endothelial barrier function. Tissue Barriers 3:e978720. doi: 10.4161/21688370.2014.978720

Rowland, R. R., and Yoo, D. (2003). Nucleolar-cytoplasmic shuttling of PRRSV nucleocapsid protein: a simple case of molecular mimicry or the complex regulation by nuclear import, nucleolar localization and nuclear export signal sequences. Virus Res. 95, 23-33. doi: 10.1016/S0168-1702(03)00161-8

Saadoun, S., Papadopoulos, M. C., Hara-Chikuma, M., and Verkman, A. S. (2005). Impairment of angiogenesis and cell migration by targeted aquaporin-1 gene disruption. Nature 434, 786-792. doi: 10.1038/nature03460

Schwab, A., Meyering, S. S., Lepene, B., Iordanskiy, S., van Hoek, M. L., Hakami, R. M., et al. (2015). Extracellular vesicles from infected cells: potential for direct pathogenesis. Front. Microbiol. 6:1132. doi: 10.3389/fmicb.2015.01132

Sewald, X., Motamedi, N., and Mothes, W. (2016). Viruses exploit the tissue physiology of the host to spread in vivo. Curr. Opin. Cell Biol. 41, 81-90. doi: 10.1016/j.ceb.2016.04.008

Shevchenko, A., Tomas, H., Havlis, J., Olsen, J. V., and Mann, M. (2006). In-gel digestion for mass spectrometric characterization of proteins and proteomes. Nat. Protoc. 1, 2856-2860. doi: 10.1038/nprot.2006.468

Silvas, J. A., Popov, V. L., Paulucci-Holthauzen, A., and Aguilar, P. V. (2016). Extracellular vesicles mediate receptor-independent transmission of novel tickborne bunyavirus. J. Virol. 90, 873-886. doi: 10.1128/JVI.02490-15

Simon, M., Johansson, C., Lundkvist, A., and Mirazimi, A. (2009). Microtubule-dependent and microtubule-independent steps in CrimeanCongo hemorrhagic fever virus replication cycle. Virology 385, 313-322. doi: 10.1016/j.virol.2008.11.020

Spencer, K. A., and Hiscox, J. A. (2006). Characterisation of the RNA binding properties of the coronavirus infectious bronchitis virus nucleocapsid protein amino-terminal region. FEBS Lett. 580, 5993-5998. doi: 10.1016/j.febslet.2006. 09.052

Surtees, R., Dowall, S. D., Shaw, A., Armstrong, S., Hewson, R., Carroll, M. W., et al. (2016). Heat shock protein 70 family members interact with Crimean-Congo hemorrhagic fever virus and hazara virus nucleocapsid proteins and perform a functional role in the nairovirus replication cycle. J. Virol. 90, 9305-9316. doi: 10.1128/JVI.00661-16

Towne, J. E., Harrod, K. S., Krane, C. M., and Menon, A. G. (2000). Decreased expression of aquaporin (AQP)1 and AQP5 in mouse lung after acute viral infection. Am. J. Respir. Cell Mol. Biol. 22, 34-44. doi: 10.1165/ajrcmb.22.1.3818

Turkina, M. V., Olofsson, A., Magnusson, K. E., Arnqvist, A., and Vikstrom, E. (2015). Helicobacter pylori vesicles carrying CagA localize in the vicinity of cellcell contacts and induce histone $\mathrm{H} 1$ binding to ATP in epithelial cells. FEMS Microbiol. Lett. 362:fnv076. doi: 10.1093/femsle/fnv076

Verkman, A. S. (2005). More than just water channels: unexpected cellular roles of aquaporins. J. Cell Sci. 118, 3225-3232. doi: 10.1242/jcs.02519

Whitehouse, C. A. (2004). Crimean-Congo hemorrhagic fever. Antiviral Res. 64, 145-160. doi: 10.1016/S0166-3542(04)00163-9

Wulan, W. N., Heydet, D., Walker, E. J., Gahan, M. E., and Ghildyal, R. (2015). Nucleocytoplasmic transport of nucleocapsid proteins of enveloped RNA viruses. Front. Microbiol. 6:553. doi: 10.3389/fmicb.2015. 00553

Yang, C., Hoelzle, M., Disanza, A., Scita, G., and Svitkina, T. (2009). Coordination of membrane and actin cytoskeleton dynamics during filopodia protrusion. PLoS ONE 4:e5678. doi: 10.1371/journal.pone.0005678

Zegers, M. M., and Friedl, P. (2014). Rho GTPases in collective cell migration. Small GTPases 5:e28997. doi: 10.4161/sgtp.28997

Zybailov, B., Mosley, A. L., Sardiu, M. E., Coleman, M. K., Florens, L., and Washburn, M. P. (2006). Statistical analysis of membrane proteome expression changes in Saccharomyces cerevisiae. J. Proteome Res. 5, 2339-2347. doi: 10.1021/pr060161n

Conflict of Interest Statement: The authors declare that the research was conducted in the absence of any commercial or financial relationships that could be construed as a potential conflict of interest.

The reviewer TF and handling Editor declared their shared affiliation, and the handling Editor states that the process nevertheless met the standards of a fair and objective review.

Copyright (C) 2017 Molinas, Turkina, Magnusson, Mirazimi and Vikström. This is an open-access article distributed under the terms of the Creative Commons Attribution License (CC BY). The use, distribution or reproduction in other forums is permitted, provided the original author(s) or licensor are credited and that the original publication in this journal is cited, in accordance with accepted academic practice. No use, distribution or reproduction is permitted which does not comply with these terms. 